UT-Komaba 02-15

hep-th/0212316

December, 2002

\title{
A New First Class Algebra, Homological Perturbation and Extension of Pure Spinor Formalism for Superstring
}

\author{
YURI AISAKA $^{\dagger}$ AND YOICHI KAZAMA $\ddagger$ \\ Institute of Physics, University of Tokyo, \\ Komaba, Meguro-ku, Tokyo 153-8902 Japan
}

\begin{abstract}
Based on a novel first class algebra, we develop an extension of the pure spinor (PS) formalism of Berkovits, in which the PS constraints are removed. By using the homological perturbation theory in an essential way, the BRST-like charge $Q$ of the conventional PS formalism is promoted to a bona fide nilpotent charge $\hat{Q}$, the cohomology of which is equivalent to the constrained cohomology of $Q$. This construction requires only a minimum number (five) of additional fermionic ghost-antighost pairs and the vertex operators for the massless modes of open string are obtained in a systematic way. Furthermore, we present a simple composite "b-ghost" field $B(z)$ which realizes the important relation $T(z)=\{\hat{Q}, B(z)\}$, with $T(z)$ the Virasoro operator, and apply it to facilitate the construction of the integrated vertex. The present formalism utilizes $U(5)$ parametrization and the manifest Lorentz covariance is yet to be achieved.
\end{abstract}

\footnotetext{
†yuri@hep1.c.u-tokyo.ac.jp

${ }^{\ddagger}$ kazama@hep1.c.u-tokyo.ac.jp
} 


\section{Introduction}

Desires to construct a quantization scheme for superstring in which both the Lorentz symmetry and the spacetime supersymmetry are manifest are ever mounting in the recent striking developments of string theory. Apart from the obvious aesthetic appeal, it should be indispensable for deeper understanding of various issues, such as the S-duality of type IIB theory and multitude of important problems involving Ramond-Ramond fields.

The conventional Ramond-Neveu-Schwarz (RNS) [1] and Green-Schwarz (GS) [2] formalisms are well-known to be inadequate for this purpose. In RNS formalism, the Lorentz symmetry is manifest but the spacetime supersymmetry is not, requiring GSO projection for its implementation. In contrast, GS formalism, which is capable of realizing both of these symmetries at the classical level, is nevertheless difficult to quantize except in the light-cone type non-covariant gauge.

About three years ago, following earlier attempts [3, 4, 15, 6, 7, 8, Berkovits initiated a new formalism [9] based on the concept of pure spinor (PS) [10, 11, 12], which is super-Poincaré covariant and posesses a number of remarkable features. The formalism is based on free fields, which form a conformal field theory (CFT) with vanishing central charge. The principal ingredient of the formalism is the BRST-like charge $Q=\int d z \lambda^{\alpha} d_{\alpha}$, where $d_{\alpha}$ is the spinor covariant derivative and $\lambda^{\alpha}$ is a bosonic chiral spinor field satisfying the pure spinor constraints $\lambda^{\alpha} \gamma_{\alpha \beta}^{\mu} \lambda^{\beta}=0$. With these constraints $Q$ becomes nilpotent and inherently second class constraints $d_{\alpha}$ can be consistently implemented as $Q \psi=0$ without losing manifest Lorentz covariance. All the perturbative physical states of superstring have been demonstrated to be realized precisely as the elements of the constrained cohomology of $Q$ [13]. Spacetime supersymmetry is manifestly maintained throughout the formalism without the need of GSO projection nor the complication due to picture changing. Covariant rules can be given with which one can compute the scattering amplitudes in a manifestly super-Poincaré covariant manner [9, 14]. Although rather involved, the relation to RNS formalism has essentially been understood [15]. This formalism can be easily applied to a superparticle [16] and an intriguing application to supermembrane has also been suggested[17]. Other recent developments are found in [18. The rudiments of this formalism will be briefly reviewed in Sec.2, while for more comprehensive review we refer the reader to [19].

Quite attractive as it is, there are many challenges for PS formalism. Essentially they all stem from the lack of understanding of the origin of the formalism. The underlying symmetry principle and the fundamental action are not known. This is reflected on the as 
yet obscure nature of the BRST-like charge, although one can relate it to RNS formalism in a round-about way 15. In this regard, recently an attempt has been made to derive the Berkovits formalism from the superembedding approach[20]. This is an interesting and aspiring endeavor but so far the analysis is completely classical and the justification of the identification of the BRST charge is not yet achieved.

Continuing along the list of to-be-improved items, we must mention the covariant rules proposed for calculations of scattering amplitudes. These rules are well-motivated and reasonable but they should be derived from more basic principles. In fact, surprisingly, to our knowledge a serious study of the inner product structure of the PS formalism has not been performed. An analysis of this issue will be presented in Sec.3, which will point to another important question about PS formalism, namely whether PS constraints are really necessary. One would like the answer to be "no" as the removal of the PS constraints should be more natural and useful in the quantization of eventual underlying action. Recently, a proposal in this direction was made in [21], where a BRST-like nilpotent charge was constructed without PS constraints in a step-wise covariant manner based on a Kac-Moody algebra with a central charge. This formalism has many desired properties but to get non-trivial cohomology one must impose an extra condition and this makes the formalism rather involved. Also an attempt to remove the PS constraints for the superparticle case has recently been reported [22]. The proposed scheme is covariant and shares some features with our present work but appears to suffer from the long-standing problem of ghosts for ghosts ad infinitum.

In this paper, we shall develop a different scheme to remove the PS constraints from the Berkovits' formalism. Our formalism is based on a novel closed first class algebra i.e. without central charges, a member of which is the BRST-like current $j(z)$ of Berkovits but now without the PS constraints. Formulation of this algebra and the subsequent construction of a nilpotent charge and vertex operators are performed in the so-called $U(5)$ parametrization, as in the original PS formalism, so that these intermediate steps are not manifestly Lorentz covariant. This is certainly a disadvantage compared to the approach of [21] but we gain considerably in the simplicity of the formalism. As it will be explained in detail in Sec.5, the construction of a new nilpotent BRST-like charge, to be called $\hat{Q}$, requires only a minimum number of anti-commuting ghost pairs $\left(c_{I}, b_{I}\right)_{I=1 \sim 5}$ and turned out to fit beautifully into the scheme of homological perturbation theory ${ }^{1}[23$ in a profound way. The biggest advantage of this scheme is that the cohomology of $\hat{Q}$ is easily shown to be exactly equivalent to the constrained cohomology of $Q$. Furthermore, the logic

\footnotetext{
${ }^{1}$ This scheme has been used in [15] in a step relating PS to RNS formalisms.
} 
of the proof of this equivalence can be applied to the systemtic construction of the vertex operators, both unintegrated and integrated, in a transparent fashion. Another important outcome of our investigation is that, in the course of the analysis of the integrated vertex operator, we discovered a remarkably simple composite " $b$-ghost" field $B(z)$, which realizes the fundamental relation $T(z)=\{\hat{Q}, B(z)\}$, where $T(z)$ is the Virasoro operator of our system. This provides an alternative far simpler method of construction for the integrated vertex. This relation to the Virasoro generator, which hitherto has been rather elusive in PS formalism, is known to be of prime importance for no-ghost theorem and loop calculations [24] and is expected to play crucial roles in future developments.

We organize the rest of this paper as follows: In Sec. 2, we start with a short review of Berkovits' pure spinor formalism, which at the same time introduces our notations and conventions. In Sec. 3, we present an analysis of the appropriate inner product in the PS formalism, with which one should implement the peculiar hermiticity property of the pure spinor. This study leads to yet another motivation for removing the PS constraints. The main results of this paper will be described starting from Sec. 4. In Sec.4, we construct a new closed first class algebra, which will be the basis of our formalism. Then, in Sec. 5, after giving a brief description of the construction of a nilpotent BRST-like charge via Batalin-Vilkovisky procedure, we apply an alternative scheme of homological perturbation theory, more natural and far-reaching in the present context, and obtain a simpler nilpotent charge $\hat{Q}$. Subsequently, we sketch the proof of the equivalence of the $\hat{Q}$-cohomology to that of the constrainted cohomology of $Q$ in the Berkovits' formalism. Having understood all the necessary fields of our formalism, we end this section by displaying the free action and the energy-momentum tensor. Sec. 6 will be devoted to the systematic construction of the physical vertex operators for the massless modes of open superstring. Both the unintegrated and the integrated vertices are obtainted in a systematic manner. Further, we present the simple composite " $b$-ghost" field $B(z)$, prove the key relation $T(z)=\{\hat{Q}, B(z)\}$ and give an alternative more superior derivation of the integrated vertex. Finally in Sec. 7, we summarize our findings and discuss remaining issues. Two appendices are provided to explain some technicalities: In Appendix A, we summarize our conventions for $\Gamma$-matrices and $U(5)$ parametrizations. Appendix $\mathrm{B}$ is devoted to the proof of the triviality of the $\delta$-homology, which forms the basis of the homological perturbation. 


\section{Rudiments of Pure Spinor Formalism}

We begin with a brief review of the essential ingredients of the pure spinor formalism, which at the same time serves to introduce our notations and conventions. Throughout we refer only to the holomorphic sector, appropriate for open string.

The central idea of the pure spinor formalism proposed by Berkovits [9] is that the physical states of superstring can be described as the elements of the cohomology of a BRST-like operator $Q$ given by ${ }^{2}$

$$
Q=\int[d z] \lambda^{\alpha}(z) d_{\alpha}(z)
$$

where $\lambda^{\alpha}$ is a 16-component bosonic chiral spinor satisfying the pure spinor constraints

$$
\lambda^{\alpha} \gamma_{\alpha \beta}^{\mu} \lambda^{\beta}=0
$$

and $d_{\alpha}$ is the spinor covariant derivative given in our convention ${ }^{3}$ by

$$
d_{\alpha}=p_{\alpha}+i \partial x_{\mu}\left(\gamma^{\mu} \theta\right)_{\alpha}+\frac{1}{2}\left(\gamma^{\mu} \theta\right)_{\alpha}\left(\theta \gamma_{\mu} \partial \theta\right)
$$

Here $x^{\mu}$ and $\theta^{\alpha}$ are, respectively, the basic bosonic and ferminonic worldsheet fields describing a superstring, which transform under the spacetime supersymmetry with global spinor parameter $\epsilon^{\alpha}$ as

$$
\delta \theta^{\alpha}=\epsilon^{\alpha}, \quad \delta x^{\mu}=i \epsilon \gamma^{\mu} \theta
$$

$x^{\mu}$ is self-conjugate and satisfies $x^{\mu}(z) x^{\nu}(w)=-\eta^{\mu \nu} \ln (z-w)$, while $p_{\alpha}$ serves as the conjugate to $\theta^{\alpha}$ in the manner $\theta^{\alpha}(z) p_{\beta}(w)=\delta_{\beta}^{\alpha} /(z-w)$. $\theta^{\alpha}$ and $p_{\alpha}$ carry conformal weights 0 and 1 respectively. With such free field operator product expansions (OPE's), $d_{\alpha}$ satisfies the following OPE with itself:

$$
d_{\alpha}(z) d_{\beta}(w)=\frac{2 i \gamma_{\alpha \beta}^{\mu} \Pi_{\mu}(w)}{z-w}
$$

where $\Pi_{\mu}$ is the basic superinvariant combination

$$
\Pi_{\mu}=\partial x_{\mu}-i \theta \gamma_{\mu} \partial \theta .
$$

\footnotetext{
${ }^{2}$ For simplicity we will use the notation $[d z] \equiv d z /(2 \pi i)$ throughout.

${ }^{3}$ Our convention, including normalization, of a number of quantities are slightly different from those often (but not invariably) used by Berkovits. Our convention has been chosen to make the description more standard and hence familiar to non-experts in this field. Some further details of our convention are described in Appendix A.
} 
Then, due to the pure spinor constraints (2.2), $Q$ is easily found to be nilpotent and the constrained cohomology of $Q$ can be defined. The basic superinvariants $d_{\alpha}, \Pi^{\mu}$ and $\partial \theta^{\alpha}$ form the closed algebra

$$
\begin{aligned}
d_{\alpha}(z) d_{\beta}(w) & =\frac{2 i \gamma_{\alpha \beta}^{\mu} \Pi_{\mu}(w)}{z-w}, \\
d_{\alpha}(z) \Pi^{\mu}(w) & =\frac{-2 i\left(\gamma^{\mu} \partial \theta\right)_{\alpha}(w)}{z-w}, \\
\Pi^{\mu}(z) \Pi^{\nu}(w) & =-\frac{\eta^{\mu \nu}}{(z-w)^{2}}, \\
d_{\alpha}(z) \partial \theta^{\beta}(w) & =\frac{\delta_{\alpha}^{\beta}}{(z-w)^{2}},
\end{aligned}
$$

which has a central charge and hence is essentially of second class. The supersymmetry transformation is generated by the supercharge 3

$$
q_{\alpha}=\int[d z]\left\{p_{\alpha}-i \partial x_{\mu}\left(\gamma^{\mu} \theta\right)_{\alpha}-\frac{1}{6}\left(\gamma^{\mu} \theta\right)_{\alpha}\left(\theta \gamma_{\mu} \partial \theta\right)\right\}
$$

which obeys the supersymmetry algebra $\left\{q_{\alpha}, q_{\beta}\right\}=-2 i \gamma_{\alpha \beta}^{\mu} \int[d z] \Pi_{\mu}(z)$.

Although eventually all the rules can be formulated in a Lorentz covariant manner, various quantities involving the pure spinor $\lambda$ are first defined transparently in the socalled $U(5)$ basis $^{4}$. As is well-known, the spinor representations for $S O(9,1)$ and $S O(10)$ can be efficiently constructed using 5 pairs of fermionic oscillator variables $\left(b_{I}, b_{I}^{\dagger}\right)$ satisfying $\left\{b_{I}, b_{J}^{\dagger}\right\}=\delta_{I J}$, which in the case of $S O(10)$ transform as $(5, \overline{5})$ of $U(5)$ subgroup $^{5}$. In this basis, to be often referred to as a U-basis in this work, a chiral spinor $\lambda$ is described by the component fields $\lambda_{A}$ given by

$$
\lambda_{A}=\left(\lambda_{+}, \lambda_{I J}, \lambda_{\tilde{I}}\right) \sim(1,10, \overline{5}) \in U(5)
$$

where we have indicated how they transform under $U(5)$, with a tilde on the $\overline{5}$ components ${ }^{6}$. In this representation, the pure spinor constraints (2.2) reduce to the 5 independent conditions

$$
\Phi_{I} \equiv \lambda_{+} \lambda_{\tilde{I}}-\frac{1}{8} \epsilon_{I J K L M} \lambda_{J K} \lambda_{K L}=0 .
$$

\footnotetext{
${ }^{4}$ Our conventions for $U(5)$ parametrization is summarized in Appendix A

${ }^{5}$ When we discuss the hermiticity property of $\lambda$ in Sec.3, the distinction between $S O(9,1)$ and $S O(10)$ becomes important. However, since the conversion between them is rather trivial for other purposes, we will use the terminology appropriate for $S O(10)$.

${ }^{6}$ In what follows, to avoid unnecessary clutter in notations, we omit tilde for $\overline{5}$ indices except when it is absolutely essential. One can easily recover the correct type of indices if needed.
} 
Therefore the number of independent components of a pure spinor is 11 and together with all the other fields (including the conjugates to the independent components of $\lambda$ ) the entire system constitutes a free CFT with vanshing central charge.

The fact that the constrained cohomology of $Q$ is in one to one correspondence with the light-cone degrees of freedom of superstring was shown in [13] using the $S O(8)$ parametrization of a pure spinor. Besides being non-covariant, this parametrization contains redundancy and an infinite number of supplimentary ghosts had to be introduced. Nonetheless, subsequently the Lorentz invariance of the cohomology was demonstrated in 25.

The great advantage of this formalism is that one can compute the scattering amplitudes under a set of rules which are manifestly super-Poincaré covariant. For the massless modes the physical unintegrated vertex operator is given by a simple form

$$
U_{0}=\lambda^{\alpha} A_{\alpha}(x, \theta)
$$

where $A_{\alpha}$ is a spinor superfield satisfying the "on-shell" condition

$$
\left(\gamma^{\mu_{1} \mu_{2} \ldots \mu_{5}}\right)^{\alpha \beta} D_{\alpha} A_{\beta}=0
$$

with

$$
D_{\alpha}=\frac{\partial}{\partial \theta^{\alpha}}-i\left(\gamma^{\mu} \theta\right)_{\alpha} \frac{\partial}{\partial x^{\mu}}
$$

Then, together with the pure spinor constraints, $Q U_{0}=0$ is easily verified and moreover $\delta U_{0}=Q \Lambda$ represents the gauge transformation of $A_{\alpha}$. Its integrated counterpart $\int[d z] V_{0}(z)$, needed for the calculation of $n$-point amplitudes with $n \geq 4$, is characterized by $Q V_{0}=\partial U_{0}$ and was constructed to be of the form [9] 3 ]

$$
V_{0}=\partial \theta^{\alpha} A_{\alpha}+\Pi^{\mu} B_{\mu}+d_{\alpha} W^{\alpha}+\frac{1}{2} L_{(\lambda)}^{\mu \nu} F_{\mu \nu}
$$

Here, $B_{\mu}=(i / 16) \gamma_{\mu}^{\alpha \beta} D_{\alpha} A_{\beta}$ is the gauge superfield, $W^{\alpha}=(i / 20)\left(\gamma^{\mu}\right)^{\alpha \beta}\left(D_{\beta} B_{\mu}-\partial_{\mu} A_{\beta}\right)$ is the gaugino superfield, $F_{\mu \nu}=\partial_{\mu} B_{\nu}-\partial_{\nu} B_{\mu}$ is the field strength superfield and $L_{(\lambda)}^{\mu \nu}$ is the Lorentz generator for the pure spinor sector. In this construction various relations [26] following solely from the on-shell condition (2.15) play crucial roles. They will be displayed later in Sec. 6 when we need them. In a similar manner, the vertex operators for the first massive modes have also been constructed recently [27].

With these vertex operators, the scattering amplitude is expressed as

$$
\mathcal{A}=\left\langle U_{1}\left(z_{1}\right) U_{2}\left(z_{2}\right) U_{3}\left(z_{3}\right) \int\left[d z_{4}\right] V_{4}\left(z_{4}\right) \cdots \int\left[d z_{N}\right] V_{N}\left(z_{N}\right)\right\rangle,
$$


and can be computed in a covariant manner with certain rules assumed for the integration over the zero modes of $\lambda^{\alpha}$ and $\theta^{\alpha}$. The proposed prescription enjoys a number of required properties and leads to results which agree with those obtained in the RNS formalism [9] 14] [15.

Sketched above are the basic ingredients of the pure spinor formalism. There have been a number of applications of this formalism, including those to a superparticle, a supermembrane, etc. For these and other related developments we refer the reader to a recent review by Berkovits [19].

\section{Hermiticity and Inner Product in Pure Spinor For- malism}

As was pointed out in the introduction, one of the important issues in PS formalism is the clarification of the Hilbert space structure, in particular the proper definition of the inner product with which the peculiar hermiticity property of the pure spinor $\lambda^{\alpha}$ should be realized in a natural manner. In this section, we shall examine this problem in some detail and find that with the PS constraints implementation of an appropriate inner product is extremely difficult if not impossible. This observation strengthens the motivation to remove the PS constraints, which will be achieved in subsequent sections.

\subsection{Hermiticity of $\lambda^{\alpha}$}

Let us begin with a description of the peculiarity of the hermiticiy property of $\lambda^{\alpha}$, which is used to construct the basic BRST-like charge $Q=\int[d z] \lambda^{\alpha}(z) d_{\alpha}(z)$. Up to a certain point, all the discussions will be valid without the PS constraints. As it will be clear, the peculiarity shows up in different guises depending on the spinor basis chosen.

First consider the usual basis, to be called a real-basis or an $R$-basis for short, in which the $S O(9,1) \gamma$-matrices are all real. Since $Q$ must be hermitian and in R-basis $d_{\alpha}(z)$, with $\gamma^{\mu}$ 's in it being real, satisfies the property $d_{\alpha}(z)^{\dagger}=z^{2} d_{\alpha}(z)$ according to the usual hermiticity property of $x^{\mu}, p_{\alpha}$ and $\theta^{\alpha}, \lambda^{\alpha}(z)$ in turn must be hermitian, namely

$$
\left(\lambda^{\alpha}(z)\right)^{\dagger}=\lambda^{\alpha}(z)
$$

On the other hand, we know that $\lambda^{\alpha}$ must be complex in order to satisfy the pure spinor conditions. This by itself is of course not inconsistent since a hermitian operator can have complex eigenvalues. However, we do anticipate some complications as the Hilbert space metric becomes necessarily indefinite and there will be null states. 
Actually, since all the basic definitions were made in $U(5)$ parametrization in Berkovits' formalism, it is better to study the problem in U-basis, where it appears in a different way 15]. To see this in more detail and to understand the nature of the issue, we should go back to the basic definition of hermiticity for spinor fields in a general basis. As a textbook matter, the charge conjugate of a spinor $\psi$ is defined by $\psi^{c}=B \psi^{*}$ and $\psi$ is real if $\psi^{c}=\psi$. Here $B$ is the matrix satisfying the properties $B \Gamma^{\mu} B^{-1}=\Gamma^{\mu *}, B^{*}=B^{T}=B, B^{2}=1$, and is related to the charge conjugation matrix $C$ by the relation $B=-C \Gamma^{0}$. This means that a hermitian spinor operator $\lambda$ should be characterized by

$$
\lambda^{\dagger}=B \lambda
$$

This of course reduces to the simple form (3.1) in an R-basis, where $B=1$. In a general basis, however, $B$ cannot be taken to be unity. This is because it does not transform by a similarity transformation under the change of spinor basis: Under a basis transformation $\tilde{\Gamma}^{\mu}=T \Gamma^{\mu} T^{-1}, B$ transforms as $\tilde{B}=T B T^{*-1}$ and this is not a similarity transformation unless $T^{*}=T$.

Let us now go to the U-basis and spell out the condition of hermiticity. In this basis, $C$ and $B$ matrices are given by $C=-\Gamma^{0} \Gamma^{2} \Gamma^{4} \Gamma^{6} \Gamma^{8}, B=-\Gamma^{2} \Gamma^{4} \Gamma^{6} \Gamma^{8}$. To write out the explicit content of (3.2), it is convenient to use the representation in terms of the fermionic oscillators $^{7}\left(b_{I}, b_{I}^{\dagger}\right)$. $B$ is then given by $B=-\left(b_{1}-b_{1}^{\dagger}\right)\left(b_{2}-b_{2}^{\dagger}\right)\left(b_{3}-b_{3}^{\dagger}\right)\left(b_{4}-b_{4}^{\dagger}\right)$, and satisfies the properties $B b_{0}^{\dagger}=b_{0}^{\dagger} B$ and $B b_{i}^{\dagger}=b_{i} B,(i=1 \sim 4)$. Applying $B$ to a chiral spinor $\lambda$, which in the oscillator representation in U-basis can be represented by a ket as

$$
|\lambda\rangle=\lambda_{+}|+\rangle+\frac{1}{2} \lambda_{I J}|I J\rangle+\lambda_{\tilde{I}}|\tilde{I}\rangle
$$

one easily finds that the hermiticity condition (3.2) amounts to the relations

$$
\begin{aligned}
\lambda_{+}^{\dagger} & =-\lambda_{\tilde{0}}, \\
\lambda_{i j}^{\dagger} & =\frac{1}{2} \epsilon_{i j k l} \lambda_{k l}, \\
\lambda_{0 i}^{\dagger} & =\lambda_{i} .
\end{aligned}
$$

Thus, the components of a hermitian chiral spinor in this basis have peculiar properties in that conjugation relates different components.

It is instructive to display briefly how the components of $\lambda$ in $\mathrm{R}$ - and $\mathrm{U}$ - bases are related. In a particular R-basis, for example, components of $\lambda^{\alpha}$ can be expressed in terms

\footnotetext{
${ }^{7}$ For more details, see Appendix A.
} 
of its U-basis components as

$$
\begin{aligned}
\lambda^{1} & =\frac{1}{2}\left(\lambda_{+}-\lambda_{\tilde{0}}-\lambda_{23}-\lambda_{14}\right), \\
\lambda^{2} & =\frac{i}{2}\left(\lambda_{12}-\lambda_{34}+\lambda_{13}+\lambda_{24}\right) \\
& \vdots \\
\lambda^{15} & =\frac{i}{2}\left(\lambda_{03}-\lambda_{\tilde{3}}+\lambda_{02}-\lambda_{\tilde{2}}\right) \\
\lambda^{16} & =\frac{1}{2}\left(\lambda_{04}+\lambda_{\tilde{4}}-\lambda_{01}-\lambda_{\tilde{1}}\right)
\end{aligned}
$$

One can easily see that they indeed satisfy $\lambda^{\alpha \dagger}=\lambda^{\alpha}$ according to the rules (3.4).

\subsection{Proper inner product}

Let us now ask how we can realize such a peculiar hermitian conjugation property in the Hilbert space of our CFT. In what follows, the usual Fock space inner product will be denoted by $(u, v)$ and the hermitian conjugation with respect to it by $u^{*}$. In a basis with non-trivial $B$, such as in a U-basis, the ordinary Fock space conjugation for the Fourier mode, like $\lambda_{n}{ }^{*}=\lambda_{-n}$, is clearly insufficient. To remedy this, we introduce a new inner product $\langle u, v\rangle$ by

$$
\langle u, v\rangle \equiv(u, S v)
$$

where $S$ is a kind of "metric" operator. Then, the hermitian conjugation of an operator $\mathcal{O}$ with respect to this new inner product is given by $\langle u, \mathcal{O} v\rangle=\left\langle\mathcal{O}^{\dagger} u, v\right\rangle$, which in the Fock space language reads $(u, S \mathcal{O} v)=\left(\mathcal{O}^{\dagger} u, S v\right)$. Now using the Fock space conjugation, $(u, S \mathcal{O} v)$ can be rewritten as $\left(\left(S^{-1}\right)^{*} \mathcal{O}^{*} S^{*} u, S v\right)$, and hence we have

$$
\mathcal{O}^{\dagger}=\left(S^{-1}\right)^{*} \mathcal{O}^{*} S^{*}
$$

We see that the new conjugation is supplemented with a similarity transformation by $S^{*}$ and can be quite non-trivial. Since $S$ must be invertible it will be convenient to write it as

$$
S=e^{-R^{*}}
$$

The new hermitian conjugation on $\mathcal{O}$ is involutive provided $\left(S^{-1} S^{*}\right)^{-1} \mathcal{O}\left(S^{-1} S^{*}\right)=\mathcal{O}$. This is satisfied if $S^{*}=S$ but it is not a necessary condition, as we shall see. 


\subsection{Explicit construction of $S$ and problem with pure spinor constraints}

Hereafter we will work in a U-basis, where all the basic properties of the operators in pure spinor formalism have actually been derived. We regard the components $\lambda_{A}$ of $\lambda$ in this basis to be the basic conformal fields satisfying the usual Fock space hermiticity $\lambda_{A}^{*}=\lambda_{A}$.

Our task now is to construct the operator $R$, which according to (3.6) effects

$$
\lambda^{\dagger}=e^{R} \lambda e^{-R}=B \lambda .
$$

Since $B$ is real, we have $\left(\lambda^{\dagger}\right)^{\dagger}=e^{R} B \lambda e^{-R}=B B \lambda=\lambda$, so this conjugation is involutive. It is useful to note that $B$ can be written as

$$
B=e^{-(i \pi / 2)(1-B)},
$$

which follows from the property $B^{2}=1$. Then, it is easy to see that (3.8) is fulfilled if $R$ satisfies

$$
[R, \lambda]=-\frac{i \pi}{2}(1-B) \lambda=-\frac{i \pi}{2}\left(\lambda-\lambda^{\dagger}\right)
$$

If we do not impose any constraints on $\lambda$, then it is quite easy to construct such an operator. Since $\lambda_{A}$ are all independent, we can introduce their conjugates $\omega_{A}$, carrying dimension 1 , which satisfy the simple OPE of the form ${ }^{8}$

$$
\lambda_{A}(z) \omega_{B}(w)=\frac{\delta_{A B}}{z-w} .
$$

Then, $R$ can be defined as

$$
R=\frac{i \pi}{2} \int[d z]: \lambda_{A}(z)(1-B)_{A B} \omega_{B}(z):,
$$

and (3.10) and hence (3.8) are realized.

On the other hand, if one imposes the PS constraints, construction becomes extremely difficult, if not impossible. Since $\lambda_{\tilde{I}}=(1 / 8) \lambda_{+}^{-1} \epsilon_{I J K L M} \lambda_{J K} \lambda_{L M}$ are now dependent composite fields, genuine conjugates to $\lambda_{A}$ do not exist. The closest analogue of $\omega_{A}$ is the field introduced in [9], denoted here by $\tilde{\omega}_{A}$, which satisfies the OPE

$$
\lambda_{A}(z) \tilde{\omega}_{B}(w)=\frac{(1-K)_{A B}}{z-w},
$$

\footnotetext{
${ }^{8}$ We follow the convention of Friedan-Martinec-Shenker 28] so that the sign is opposite to that of Berkovits.
} 
with $K_{A B}$ a projector needed for consistency with PS constrains. Although $\tilde{\omega}$ and $K$ have a number of nice properties and are extremely useful in the pure spinor formalism, one cannot simply substitute $\tilde{\omega}_{B}$ in place of $\omega_{B}$ in (3.12). In fact the problem appears to be rather serious. Consider for example the equation (3.10) for the component $\lambda_{\tilde{i}}$. Since $\left(\lambda_{\tilde{i}}\right)^{\dagger}=\lambda_{0 i}$, we must have

$$
\left[R, \lambda_{\tilde{i}}\right]=-\frac{i \pi}{2}\left(\lambda_{\tilde{i}}-\lambda_{0 i}\right)
$$

But since $\lambda_{\tilde{i}}$ is already cubic in the basic fields, it is practically impossible from the commutator to produce the term $\lambda_{0 i}$ on the RHS, which is linear (or quadratic in some parametrization [9]) in the independent fields. We have not been able to construct $R$ which effects the proper transformation (3.10) for all the components consistently.

This strongly indicates that in order to define an appropriate inner product with which one can properly implement the hermiticity property and compute the amplitudes from the first principle, it appears to be imperative to remove the pure spinor constraints. In the remainder of this paper, we will present a rather elegant way of achieving this.

\section{A New Closed First Class Algebra}

Besides the ones mentioned in the introduction, the analysis of the previous section added another reason to try to remove the PS constraints. In this and the subsequent sections, we shall show that it is indeed possible to achieve this by constructing a BRST-like charge, to be called $\hat{Q}$, which is nilpotent without the PS constraints and who's cohomology is identical to the constrained cohomology of $Q$. We begin, in this section, by demonstrating that out of the system of second class constraints formed by the basic operators $d_{\alpha}, \Pi^{\mu}$ and $\partial \theta^{\alpha}$ we can rather naturally construct, by using $U(5)$ formalism, a new closed first class algebra.

\section{1 $U(5)$ decompositions}

For this purpose, we need to develop some tools to facilitate the manipulations in $U(5)$ basis. Let $u^{\mu}$ be an $S O(9,1)$ vector. In the following, we will write $u^{0}=i u^{10}$ and use $S O(10)$ notation. Hence the upper and the lower indices will at times not be distinguished.

Now $u^{\mu}$ can be decomposed into two sets of 5 -vectors $u_{I}^{+}$and $u_{I}^{-},(I=1 \sim 5)$, which are 5 and $\overline{5}$ of $U(5)$ respectively. This is done by introducing the "intertwiners" $e_{I}^{ \pm \mu}$ as

$$
u_{I}^{ \pm}=e_{I}^{ \pm \mu} u_{\mu}
$$


where

$$
e_{I}^{ \pm \mu} \equiv \frac{1}{2}\left(\delta_{\mu, 2 I-1} \pm i \delta_{\mu, 2 I}\right)
$$

It is easy to see that $e_{I}^{ \pm \mu}$ enjoy the following basic properties:

$$
\begin{aligned}
& e_{I}^{ \pm \mu} e_{J}^{ \pm \mu}=0, \quad e_{I}^{ \pm \mu} e_{J}^{\mp \mu}=\frac{1}{2} \delta_{I J}, \\
& e_{I}^{+\mu} e_{I}^{-\nu}+e_{I}^{-\mu} e_{I}^{+\nu}=\frac{1}{2} \delta^{\mu \nu}
\end{aligned}
$$

Using the last of these properties, $u^{\mu}$ can be expanded as

$$
u^{\mu}=2\left(e_{I}^{+\mu} u_{I}^{-}+e_{I}^{-\mu} u_{I}^{+}\right)
$$

In particular, the $\Gamma$-matrices can be decomposed as

$$
\Gamma^{\mu}=2\left(e_{I}^{+\mu} \Gamma_{I}^{-}+e_{I}^{-\mu} \Gamma_{I}^{+}\right)
$$

where $\Gamma_{I}^{ \pm}$are nothing but the fermionic oscillators $b_{I}$ and $b_{I}^{\dagger}$ :

$$
\Gamma_{I}^{+}=e_{I}^{+\mu} \Gamma^{\mu}=b_{I}, \quad \Gamma_{I}^{-}=e_{I}^{-\mu} \Gamma^{\mu}=b_{I}^{\dagger} .
$$

Next, we wish to express spinor bilinears in $U(5)$ basis. Following the method described in Appendix A, one can easily work out the formulas such as

$$
\begin{aligned}
e_{I}^{+\mu}\left(\lambda \gamma^{\mu} \chi\right) & =-\left(\lambda_{\tilde{J}} \chi_{I J}+\lambda_{I J} \chi_{\tilde{J}}\right) \\
e_{I}^{-\mu}\left(\lambda \gamma^{\mu} \chi\right) & =-\left(\lambda_{+} \chi_{\tilde{I}}+\lambda_{\tilde{I}} \chi_{+}\right)+\frac{1}{4} \epsilon_{I J K L M} \lambda_{J K} \chi_{L M}
\end{aligned}
$$

where $\lambda$ and $\chi$ are both chiral.

Now we come to a simple but important observation. Let $\lambda^{\alpha}$ be a bosonic chiral spinor and define

$$
\Lambda^{\mu} \equiv \lambda \gamma^{\mu} \lambda
$$

10 conditions $\Lambda^{\mu}=0$ make $\lambda^{\alpha}$ to be a pure spinor, which actually has 11 independent components. This means that half of the conditions must be redundant. To see this more explicitly, decompose $\Lambda^{\mu}$ according to the formula (4.8) and (4.9). We get

$$
\begin{aligned}
& \Lambda_{I}^{+}=-2 \lambda_{I J} \lambda_{\tilde{J}}, \\
& \Lambda_{I}^{-}=-2 \Phi_{I},
\end{aligned}
$$


where

$$
\begin{aligned}
\Phi_{I} & \equiv \lambda_{+} \lambda_{\tilde{I}}-\frac{1}{8} E_{I} \\
E_{I} & \equiv \epsilon_{I J K L M} \lambda_{J K} \lambda_{L M} .
\end{aligned}
$$

Evidently $\Lambda_{I}^{-}$is directly proportional to the genuinely independent pure spinor constraint $\Phi_{I}$ but $\Lambda_{I}^{+}$is not. However, by using (4.13) we can rewrite it as ${ }^{9}$

$$
\Lambda_{I}^{+}=-2 \lambda_{+}^{-1} \lambda_{I J} \Phi_{J}-\frac{1}{4} \lambda_{+}^{-1} \lambda_{I J} E_{J}
$$

Now by a simple yet slightly non-trivial identity, the second term on the RHS vanishes and we find that $\Lambda_{I}^{+}$is also a linear comibination of $\Phi_{I}$. Summarizing, we find that $\Lambda^{\mu}$ can be decomposed naturally as

$$
\Lambda^{\mu}=\mathcal{N}_{I}^{\mu} \Phi_{I}
$$

where

$$
\mathcal{N}_{I}^{\mu} \equiv-4\left(e_{I}^{+\mu}-\lambda_{+}^{-1} \lambda_{I J} e_{J}^{-\mu}\right)
$$

are a set of 5 vectors. Moreover it is easy to check that they form a system of 5 independent mutually orthogonal null vectors, consistent with the fact that $\Lambda^{\mu}$ itself is null due to the well-known Fierz identity

$$
\left(\gamma^{\mu}\right)_{\alpha \beta}\left(\gamma_{\mu}\right)_{\gamma \delta}+\text { cyclic in }(\alpha, \beta, \gamma)=0
$$

In fact, there exists another natural set of null vectors defined by

$$
\overline{\mathcal{N}}_{I}^{\mu} \equiv-\frac{1}{2} e_{I}^{-\mu}
$$

$\overline{\mathcal{N}}_{I}^{\mu}$ are complimentary to $\mathcal{N}_{I}^{\mu}$ and they together satisfy the following orthonormality and completeness relations:

$$
\begin{aligned}
\mathcal{N}_{I}^{\mu} \mathcal{N}_{J}^{\mu} & =0, \quad \overline{\mathcal{N}}_{I}^{\mu} \overline{\mathcal{N}}_{J}^{\mu}=0, \\
\mathcal{N}_{I}^{\mu} \overline{\mathcal{N}}_{J}^{\mu} & =\delta_{I J}, \\
\mathcal{N}_{I}^{\mu} \overline{\mathcal{N}}_{I}^{\nu}+\mathcal{N}_{I}^{\nu} \overline{\mathcal{N}}_{I}^{\mu} & =\delta^{\mu \nu} .
\end{aligned}
$$

These null vectors will play important roles $^{10}$.

\footnotetext{
${ }^{9}$ We assume $\lambda_{+} \neq 0$, as in the original Berkovits formalism.

${ }^{10}$ This is suspected to be deeply related to the fact that pure spinors originally arose in the description of null-planes [10]. It would be interesting to uncover the geometrical significance of our formalism.
} 


\subsection{New first class algebra}

Let us recall the set of OPE's among the basic superinvariant operators of dimension 1:

$$
\begin{aligned}
d_{\alpha}(z) d_{\beta}(w) & =\frac{2 i \gamma_{\alpha \beta}^{\mu} \Pi_{\mu}(w)}{z-w}, \\
d_{\alpha}(z) \Pi^{\mu}(w) & =\frac{-2 i\left(\gamma^{\mu} \partial \theta\right)_{\alpha}(w)}{z-w} \\
\Pi^{\mu}(z) \Pi^{\nu}(w) & =-\frac{\eta^{\mu \nu}}{(z-w)^{2}}, \\
d_{\alpha}(z) \partial \theta^{\beta}(w) & =\frac{\delta_{\alpha}^{\beta}}{(z-w)^{2}} .
\end{aligned}
$$

The fact that the unit operators appear on the RHS of the last two OPE's signify that they are of second class. With the help of the bosonic chiral spinor variable $\lambda^{\alpha}(z)$ and the decomposition (4.16) involving null vectors, we can now turn this system into a first class algebra without imposing any constraints on $\lambda^{\alpha}$.

First consider the OPE of the BRST current $j(z)=\lambda^{\alpha}(z) d_{\alpha}(z)$ with itself. Using (4.23) we immediately get

$$
j(z) j(w)=\frac{2 i \Lambda^{\mu} \Pi_{\mu}(w)}{z-w} .
$$

Using the decomposition (4.16) and introducing a new operator

$$
\mathcal{P}_{I} \equiv \mathcal{N}_{I}^{\mu} \Pi_{\mu}
$$

this can be written as

$$
j(z) j(w)=\frac{2 i \mathcal{P}_{I} \Phi_{I}(w)}{z-w} .
$$

Now in contrast to the origial $\Pi^{\mu}$, the operator $\mathcal{P}_{I}$ has a first class OPE with itself due to the contraction with the null vector field $\mathcal{N}_{I}^{\mu}$. Indeed, we have

$$
\begin{aligned}
\mathcal{P}_{I}(z) \mathcal{P}_{J}(w) & =\mathcal{N}_{I}^{\mu}(z) \mathcal{N}_{J}^{\nu}(w) \Pi_{\mu}(z) \Pi_{\nu}(w) \\
& =-\frac{\mathcal{N}_{I}^{\mu}(z) \mathcal{N}_{J}^{\mu}(w)}{(z-w)^{2}}=\frac{\mathcal{S}_{I J}(w)}{z-w}
\end{aligned}
$$

where the null nature of $\mathcal{N}_{I}^{\mu}$ is crucial for the disappearance of the double pole and

$$
\mathcal{S}_{I J} \equiv-\left(\partial \mathcal{N}_{I}^{\mu}\right) \mathcal{N}_{J}^{\mu}
$$

Note that $S_{I J}$ is properly antisymmetric again due to the null property of $\mathcal{N}_{I}^{\mu}$. 
Consider next the $\mathrm{OPE} j(z) \mathcal{P}(w)$. We get

$$
j(z) \mathcal{P}_{I}(w)=\lambda^{\alpha} \mathcal{N}_{I}^{\mu} d_{\alpha}(z) \Pi_{\mu}(w)=\frac{-2 i \mathcal{N}_{I}^{\mu}\left(\lambda \gamma_{\mu} \partial \theta\right)}{z-w}
$$

Now we note the following useful representation of $\mathcal{N}_{I}^{\mu}$ :

$$
\mathcal{N}_{I}^{\mu}=-2 \lambda_{+}^{-1}\left(\gamma^{\mu} \lambda\right)_{I}
$$

where $\left(\gamma^{\mu} \lambda\right)_{I} \equiv\left\langle I\left|\Gamma^{\mu}\right| \lambda\right\rangle$. Then, using the Fierz identity (4.18) one easily finds $\mathcal{N}_{I}^{\mu}\left(\lambda \gamma_{\mu} \partial \theta\right)=$ $\lambda_{+}^{-1} \Lambda^{\mu}\left(\gamma_{\mu} \partial \theta\right)_{I}=\lambda_{+}^{-1} \mathcal{N}_{J}^{\mu}\left(\gamma_{\mu} \partial \theta\right)_{I} \Phi_{J}$. Further, it is not difficult to show that $\mathcal{N}_{J}^{\mu}\left(\gamma_{\mu} \partial \theta\right)_{I}$ is actually anti-symmetric in $(I, J)$. Hence, we get

$$
j(z) \mathcal{P}_{I}(w)=\frac{\mathcal{R}_{I J} \Phi_{J}(w)}{z-w},
$$

where

$$
\mathcal{R}_{I J}=2 i \lambda_{+}^{-1} \mathcal{N}_{I}^{\mu}\left(\gamma_{\mu} \partial \theta\right)_{J}
$$

In a similar manner, the rest of OPE's between all the fields $j, \mathcal{P}_{I}, \mathcal{R}_{I J}$ and $S_{I J}$ can be computed easily and we find that altogether they form the following set of Jacobiconsistent closed first class algebra:

$$
\begin{aligned}
j(z) j(w) & =\frac{2 i \mathcal{P}_{I} \Phi_{I}}{z-w}, \\
j(z) \mathcal{P}_{I}(w) & =\frac{\mathcal{R}_{I J} \Phi_{J}}{z-w}, \\
j(z) \mathcal{R}_{I J}(w) & =\frac{-i \mathcal{S}_{I J}}{z-w}, \\
\mathcal{P}_{I}(z) \mathcal{P}_{J}(w) & =\frac{\mathcal{S}_{I J}}{z-w}, \\
\text { all the rest } & =\text { non-singular. }
\end{aligned}
$$

So with the help of unconstrained $\lambda^{\alpha}$, we have been able to turn the system with second class constraints into one which is of purely first class.

Let us make some remarks. First, an algebra similar in spirit to the above appeared in the analysis of BRST cohomology in $S O(8)$ framework [13]. The system itself was much simpler, consisting of two operators called $G^{a}$ and $T$, but as the $S O(8)$ parametrization of the PS constraints is highly redundant, these operators were quite complicated containing inifinite number of ghosts. In our case, as we have captured the content of the PS constraints without redundancy, the operators are simple with no ghosts required. Second, the method we have developed can be applied to more general systems of second class algebra. Such applications may be useful in many other contexts and will be described in a separate publication [29]. 


\section{Homological Perturbation, Nilpotent BRST-like Charge and its Cohomology}

\subsection{Batalin-Vilkovisky procedure}

In the previous section, we have obtained in a rather natural manner a new closed first class algebra. An immediate thought which comes to one's mind is to apply the usual BRST formalism of Batalin and Vilkovisky (BV) [30] to construct a nilpotent BRST-like charge associated with this algebra.

This can indeed be done, albeit with a slight peculiarity. The peculiarity is that the operators which form the algebra are not the usual constraints which generate the underlying classical gauge symmetry ${ }^{11}$. In particular, they include the current $j(z)$, which if $\lambda^{\alpha}$ were a pure spinor is interpreted as the nilpotent BRST-like current of the system. Nevertheless, BV procedure can be applied as a formal algorithmic device to construct a fermionic charge, to be called $Q^{\prime}$, which is nilpotent without pure spinor constraints. As usual we first introduce the ghost-antighost pair for each operator forming the algebra in the following way ${ }^{12}$ :

$$
\begin{array}{rll}
j & : & (\gamma, \beta), \\
\mathcal{P}_{I}: & \left(c_{I}, b_{I}\right), \\
\mathcal{R}_{I J}: & \left(\gamma_{I J}, \beta_{I J}\right), \\
\mathcal{S}_{I J}: & \left(c_{I J}, b_{I J}\right) .
\end{array}
$$

Here $(\gamma, \beta)$ ghosts are bosonic and $(c, b)$ pairs are fermionic, both carrying the conformal weights $(0,1)$, with the OPE of the form

$$
\begin{aligned}
& b_{I}(z) c_{J}(w)=\frac{\delta_{I J}}{z-w}, \\
& \beta_{I J}(z) \gamma_{K L}=-\frac{\delta_{K L}^{I J}}{z-w} \quad \text { etc. }
\end{aligned}
$$

As for the central charge counting, $\left(\gamma_{I J}, \beta_{I J}\right)$ and $\left(c_{I J}, b_{I J}\right)$ compensate, while $\left(c_{I}, b_{I}\right)$ precisely kill the contribution from the extra 5 components in $\lambda^{\alpha}$ (and their conjugates) now alive in the absence of the PS constraints. One might worry that the contribution from $(\gamma, \beta)$ ghosts remains uncancelled. However, because $j(z)$ does not appear on the RHS of the OPE's, $\beta$ will be absent in $Q^{\prime}$ and hence $\gamma$ can be simply set to 1 . Therefore the total central charge still vanishes and we have a viable free conformal field theory.

\footnotetext{
${ }^{11}$ In fact this is the reason why we have been careful to avoid calling it a "constraint" algebra.

${ }^{12}$ Notation for the anti-ghost $b_{I}$ coincides with the fermionic oscillator used to in $U(5)$ formalism, but there should be no confusion.
} 
Following the standard BV prescription, the BRST charge $Q^{\prime}$ is now constructed as $Q^{\prime}=\int[d z] j^{\prime}(z)$, where the BRST current is given by

$$
\begin{aligned}
j^{\prime}=j & +c_{I} \mathcal{P}_{I}+\frac{1}{2} \gamma_{I J} \mathcal{R}_{I J}+\frac{1}{2} c_{I J} \mathcal{S}_{I J} \\
& -i b_{I} \Phi_{I}+\beta_{I J} \Phi_{J} c_{I}+\frac{i}{2} b_{I J} \gamma_{I J}+\frac{1}{2} b_{I J} c_{I} c_{J} .
\end{aligned}
$$

It is easy to check that $Q^{\prime}$ is indeed nilpotent.

The crucial question of course is whether the cohomology of $Q^{\prime}$ is isomorphic to the constrained cohomology of $Q$. The answer turned out to be yes, but we will not give the details of the proof here. The reason is that, as we shall shortly describe, there exists an alternative scheme of producing a nilpotent BRST-like charge which is much more natural and profound in the present context than the BV procedure. It is known under the name of homological perturbation [23].

Nevertheless, it may be instructive to mention briefly how we were led to the use of the homological perturbation scheme. In the effort to demonstrate the equivalence of the aforementioned cohomologies, we first proved that any expressions involving $\left(\gamma_{I J}, \beta_{I J}\right)$ and $\left(c_{I J}, b_{I J}\right)$ ghosts are actually $Q^{\prime}$-exact and hence can be dropped. This is quite natural as these ghosts form quartets. This means that effectively $Q^{\prime}$ can be reduced to a much simpler $\tilde{Q}$ given by

$$
\tilde{Q}=Q+\delta+d_{1}
$$

where

$$
\begin{aligned}
\delta & \equiv \int[d z]\left(-i \Phi_{I} b_{I}\right), \\
d_{1} & \equiv \int[d z] c_{I} \mathcal{P}_{I} .
\end{aligned}
$$

The notations here are designed to imply that $\delta, Q$ and $d_{1}$ can be regarded as $-1,0$ and 1 form operators respectively. As we have truncated the full $Q^{\prime},\{\tilde{Q}, \tilde{Q}\}$ no longer vanishes but equals $\int[d z]\left(-2 c_{I} R_{I J} \Phi_{J}+c_{I} c_{J} S_{I J}\right)$. However, this quantity commutes with any expressions composed of $\lambda^{\alpha}, x^{\mu}, \theta^{\alpha}, c_{I}$ and their worldsheet derivatives, which are the building blocks of the unintegrated vertex operators. Moreover the pieces neglected in $Q^{\prime}$ have no singularities with such operators. Thus the cohomology analysis can indeed be carried out with $\tilde{Q}$ and in this way we succeeded in producing a proof.

At this point an alert reader may have noticed that the form of $\tilde{Q}$ is precisely the beginning of the homological perturbation scheme, which allows one to upgrade $\tilde{Q}$ into a genuinely nilpotent operator $\hat{Q}$ under appropriate conditions. Moreover the general 
theory guarantees that the cohomology of $\hat{Q}$ is equivalent to the cohomology of $Q$ with the constraint $\delta=0$, which in our case is nothing but the imposition of PS constraints. In other words, while BV procedure provides one way of nilpotent completion of $Q$, the homological perturbation, to be described below, gives another, which in our case is more natural and powerful. As we shall see, this in general leads to a completion different from the one obtained by the BV method.

\subsection{Homological perturbation and nilpotent charge $\hat{Q}$}

Now let us explain the homological perturbation scheme, as applied to our system. Since the general theory is lucidly described in [23], we shall limit the exposition to the extent necessary for our purposes, which includes the application to the construction of physical vertex operators described in Sec.6.

As was already mentioned, we will use the terminology of the differential form, where the degree of the form is defined as the total $\left(c_{I}, b_{I}\right)$ ghost number, with $\operatorname{gh} \#\left(c_{I}\right)=$ $1, \operatorname{gh} \#\left(b_{I}\right)=-1$. Also, in the following, a product $A B$ will always signify the operator product in the sense of conformal field theory. In particular, when $A$ or $B$ is an integrated operator, $A B$ equals the graded commutator $[A, B\}$. In this notation, the graded Jacobi identity reads $A B C \equiv A(B C)=(A B) C \pm B(A C)$.

Now in the homological perturbation theory, the operator $\delta$, defined in our case in (5.9), of degree -1 will play the key role. It must satisfy the following basic properties:

$$
\begin{aligned}
& \text { (i) } \quad \delta^{2}=0, \\
& \text { (ii) } \quad \delta A=0 \Rightarrow A=\delta B,
\end{aligned}
$$

where $A$ and $B$ are, respectively, an $n$-form and an $(n+1)$-form with $n \geq 1$. The property $(i)$, which in our case is obvious from the definition of $\delta$, means that one can consider the $\delta$-homology defined by $H_{n}(\delta)=\operatorname{Ker} \delta / \operatorname{Im} \delta$, where $n$ is the degree of the space on which $\delta$ acts. Then the property $(i i)$ simply states that $H_{n}(\delta)=0$ for $n \geq 1$, namely the homology is trivial above degree 1 . This property is absolutely crucial for the whole scheme to work and hence we shall give the proof for our $\delta$.

For the simple case of operators consisting of $x^{\mu}, \theta^{\alpha}, \lambda^{\alpha}$ and their worldsheet derivatives together with $c_{I}$ 's without any $\partial$, the proof is straightforward since in such a situation the action of $\delta$ is simply to replace one $c_{I}$ by $\Phi_{I}$. Let $A=c_{I_{1}} c_{I_{2}} \cdots c_{I_{n}} A_{I_{1} I_{2} \ldots I_{n}}$ be an $n$-form. Acting $\delta$, we get $\delta A=-i n c_{I_{2}} c_{I_{3}} \cdots c_{I_{n}} \Phi_{I_{1}} A_{I_{1} I_{2} I_{3} \ldots I_{n}}$. Because we have captured the PS constraints without redundancy $\Phi_{I}$ 's are algebraically independent. There- 
fore for $\delta A_{n}$ to vanish we must have $A_{I_{1} I_{2} I_{3} \ldots I_{n}}=\Phi_{J} \tilde{A}_{J I_{1} I_{2} \ldots I_{n}}$, where $\tilde{A}$ is totally antisymmetric. But then $A=\Phi_{J} c_{I_{1}} c_{I_{2}} \cdots c_{I_{n}} \tilde{A}_{J I_{1} I_{2} \ldots I_{n}}$ can be written as $\delta B$, where $B=$ $(i /(n+1)) c_{J} c_{I_{1}} c_{I_{2}} \cdots c_{I_{n}} \tilde{A}_{J I_{1} I_{2} \ldots I_{n}}$.

The proof for the general case is considerably more involved. The reason is that, in addition to the aformentioned building blocks, we may have $b_{I}, \omega_{\alpha}$, worldsheet derivatives thereof and $\partial^{m} c_{I}$ 's. The OPE of $\delta$ with such operators can produce higher order poles as well as structures other than $\Phi_{I}$ 's. Nevertheless by appropriate use of mathematical inductions the proof can be produced, which is given in Appendix B.

Besides $\delta$, another operator of prime importance is, of course, the operator $Q$. Clearly $Q$ satisfies

$$
\delta Q=0 .
$$

In other words, $Q$ anticommutes with $\delta$. Another important property of $Q$ is that while $Q^{2}$ does not vanish it is nevertheless $\delta$-exact. Indeed,

$$
Q^{2}=\int[d z] 2 i \mathcal{P}_{I} \Phi_{I}=-2 \delta d_{1},
$$

where $d_{1}$ is given in (5.10). In homological perturbation theory, $Q$ satisfying (5.13) and (5.14) is said to be a differential modulo $\delta$.

Now with this setting one can construct a nilpotent operator $\hat{Q}$ in the manner

$$
\hat{Q}=\delta+Q+d_{1}+d_{2}+\cdots,
$$

where $d_{n}$ is an operator of degree $n$. Moreover, the main theorem of homological perturbation theory states [23] that the cohomology of $\hat{Q}$ coincides with the $\delta$-constrained cohomology of $Q$.

To find $d_{n}$, we first write down the requirement of nilpotency of $\hat{Q}$ more explicitly:

$$
0=\hat{Q}^{2}=Q^{2}+2 \sum_{n \geq 1} \delta d_{n}+2 \sum_{n \geq 1} Q d_{n}+\sum_{k, l \geq 1} d_{k} d_{l} .
$$

Rather than repeating the general recursive procedure given in 23, let us see how this equation determines $d_{n}$ explicitly in our context. At overall degree 0 , the nilpotency is fulfilled due to (5.14). At degree 1, the condition becomes

$$
0=\delta d_{2}+Q d_{1}
$$

Now apply $\delta$ on the second term $Q d_{1}$. Using (15.13), a graded Jacobi identity and (5.14), we get $\delta\left(Q d_{1}\right)=-Q\left(\delta d_{1}\right)=\frac{1}{2} Q^{3}=0$. From (5.12) this means that $Q d_{1}$ must be of the 
form $Q d_{1}=-\delta X_{2}$, where $X_{2}$ is a 2-form. Then, (5.17) becomes $0=\delta\left(d_{2}-X_{2}\right)$ and this is solved for $d_{2}$ as $d_{2}=X_{2}+\delta Y_{3}$ with an arbitrary 3 -form $Y_{3}$. Note that all the manipulations are independent of the details of the operators. The explicit content of the operators does matter when we actually determine the form of $X_{2}$ etc. In our case, we have

$$
\begin{aligned}
Q d_{1} & =-\int[d z] c_{I} R_{I J} \Phi_{J}=-\delta X_{2} \\
X_{2} & =-\frac{i}{2} \int[d z] c_{I} c_{J} R_{I J} .
\end{aligned}
$$

Let us go one more step to the degree 2 analysis. The nilpotency condition now reads

$$
0=2 \delta d_{3}+2 Q d_{2}+d_{1}^{2} .
$$

Explicit calculation immediately gives $2 Q X_{2}=-c_{I} c_{J} \mathcal{S}_{I J}$ and $d_{1}^{2}=c_{I} c_{J} \mathcal{S}_{I J}$. Therefore the main part of $Q d_{2}+d_{1}^{2}$ vanishes and we are left with $\delta\left(d_{3}-Q Y_{3}\right)=0$. But since $Y_{3}$ is arbitrary, we may set it to zero and this gives $d_{3}=0$ as a viable solution. In this way, for the system at hand the perturbation terminates at this stage. Summarizing, we now have constructed a nilpotent operator $\hat{Q}$ in the form

$$
\hat{Q}=\delta+Q+d_{1}+d_{2},
$$

where $\delta, Q, d_{1}$ were already given before and $d_{2}$ can be taken to be

$$
d_{2}=-\frac{i}{2} \int[d z] c_{I} c_{J} R_{I J} .
$$

At this point, let us introduce "semi-covariant" notations for the ghosts. Define $c^{\mu}$ and $b^{\mu}$ by

$$
c^{\mu} \equiv c_{I} \mathcal{N}_{I}^{\mu}, \quad b^{\mu} \equiv b_{I} \overline{\mathcal{N}}_{I}^{\mu} .
$$

Using the orthonormality relations for $\left\{\mathcal{N}_{I}^{\mu}, \overline{\mathcal{N}}_{I}^{\mu}\right\}$ these relations can be inverted as

$$
c_{I}=c^{\mu} \overline{\mathcal{N}}_{I}^{\mu}, \quad b_{I}=b^{\mu} \mathcal{N}_{I}^{\mu} .
$$

They satisfy the OPE

$$
b^{\mu}(z) c^{\nu}(w)=\frac{\overline{\mathcal{N}}_{I}^{\mu} \mathcal{N}_{I}^{\nu}(w)}{z-w}=\frac{\eta^{\mu \nu}-\overline{\mathcal{N}}_{I}^{\nu} \mathcal{N}_{I}^{\mu}(w)}{z-w} .
$$

We emphasize that $b^{\mu}$ and $c^{\mu}$ are not genuine vector fields and this fact is reflected in this OPE. Nevertheless, for computational purposes this semi-covariant notation will be 
useful. In this notation, the operators $\delta, d_{1}$ and $d_{2}$ take simple forms:

$$
\begin{aligned}
\delta & =-\int[d z] i b^{\mu} \Lambda_{\mu}, \quad d_{1}=\int[d z] c^{\mu} \Pi_{\mu}, \\
d_{2} & =\int[d z] \lambda_{+}^{-1} c^{\mu} c^{\nu} \overline{\mathcal{N}}_{I}^{\nu}\left(\gamma_{\mu} \partial \theta\right)_{I}
\end{aligned}
$$

Some remarks are in order. (1) The scheme of homological perturbation can be thought of as a device to implement the constraints $\Phi_{I}=0$ in $\lambda$-space by enlarging the space to include $\left(c_{I}, b_{I}\right)$ ghosts, in contrast to the usual BV procedure which is normally used to implement the original gauge constraints. (2) The ambiguity such as the choice of $Y_{3}$ above can be utilized to obtain different form of $\hat{Q}$ if one desires. (3) With our simplest choice of fixing this ambiguity, only a minimum number of ghosts $\left(c_{I}, b_{I}\right)$ are needed.

\subsection{Equivalence of cohomologies}

Although the equivalence of the cohomology of $\hat{Q}$ to the constrained cohomology of $Q$ is guaranteed by the general theory of homological perturbation [23], we shall give a sketch of the proof, as the similar logic will be needed in the construction of the vertex operators.

In the proof, the nilpotency of $\hat{Q}$ will play an important role. By sorting out the equation $\hat{Q}^{2}=0$ (5.16) according to the degree, we have, in our case, the explicit relations

$$
\begin{aligned}
\delta^{2} & =0, \\
\delta Q & =0, \\
Q^{2}+2 \delta d_{1} & =0, \\
Q d_{1}+\delta d_{2} & =0, \\
d_{1}^{2}+2 Q d_{2} & =0, \\
d_{1} d_{2} & =0, \\
d_{2}^{2} & =0 .
\end{aligned}
$$

It is also useful to write down the action of $\hat{Q}$ on a general operator $X$, which can be expanded with respect to the degree ${ }^{13}$ as $X=\sum_{n=0}^{5} X_{n}$ :

$$
\hat{Q} X=\sum_{n}\left(Q X_{n}+d_{1} X_{n-1}+d_{2} X_{n-2}+\delta X_{n+1}\right) .
$$

Now we begin with the proof of $Q$-closed $\Rightarrow \hat{Q}$-closed. Let $U_{0}$ be an operator which is $Q$-closed up to the PS constraint, i.e. $Q U_{0}=U_{I} \Phi_{I}$ for some $U_{I}$. Since the RHS can

\footnotetext{
${ }^{13}$ Since $c_{I}$ has 5 components, the highest possible degree is 5 .
} 
be rewritten as $-\delta U_{1}$, where $U_{1}=-i c_{I} U_{I}$, we have $Q U_{0}+\delta U_{1}=0$, which, according to (5.34) is the $\hat{Q}$ closedness relation at degree 0 . Of course $U_{1}$ is determined only up to some $\delta X$, but such a freedom is easily seen to correspond precisely to that of adding a $\hat{Q}$-exact form and hence will be ignored hereafter. Suppose that we have constructed $U_{k}$ 's up to $k=n+1$, which satisfy the $\hat{Q}$-closedness relation

$$
Q U_{n}+d_{1} U_{n-1}+d_{2} U_{n-2}+\delta U_{n+1}=0
$$

Our task is to construct $U_{n+2}$ which satisfies the similar equation at one degree higher. Consider the expression $Y_{n+1} \equiv Q U_{n+1}+d_{1} U_{n}+d_{2} U_{n-1}$. Act $\delta$ onto this and make use of the relation (5.28). We get

$$
\delta Y_{n+1}=-Q\left(\delta U_{n+1}\right)+\delta\left(d_{1} U_{n}\right)+\delta\left(d_{2} U_{n-1}\right) .
$$

Applying the relation (5.35) to the first term on the RHS and using Jacobi identities such as $\delta\left(d_{1} U_{n}\right)=\left(\delta d_{1}\right) U_{n}-d_{1}\left(\delta U_{n}\right), Q\left(Q U_{n}\right)=\frac{1}{2} Q^{2} U_{n}$, etc., this can be rewritten into

$$
\delta Y_{n+1}=-d_{1}\left(Q U_{n-1}+d_{1} U_{n-2}+\delta U_{n}\right)-d_{2}\left(Q U_{n-2}+\delta U_{n-1}\right) .
$$

Now substituting the relations (5.35) at lower degrees, this becomes

$$
\delta Y_{n+1}=d_{1}\left(d_{2} U_{n-3}\right)+d_{2}\left(d_{1} U_{n-3}+d_{2} U_{n-4}\right)
$$

and this is seen to vanish by (5.32) and (5.33). This means that $Y_{n+1}$ must be of the structure $Y_{n+1}=-\delta U_{n+2}$ and in this way we can construct $U_{n+2}$ that satisfies the $\hat{Q}_{\text {- }}$ closedness condition at degree $n+2$. Hence $U=\sum_{n} U_{n}$ is $\hat{Q}$-closed and the proof is complete.

The logic for proving $Q$-exact $\Rightarrow \hat{Q}$-exact is a slight variation of the above. Suppose $U_{0}$ is $Q$-exact, i.e. $U_{0}=Q \Omega_{0}+\delta \Omega_{1}$ for some $\Omega_{0}$ and $\Omega_{1}$. Applying $Q$ to this relation, one easily gets $Q U_{0}=-\delta\left(Q \Omega_{1}+d_{1} \Omega_{0}\right)$. Comparing this with (5.35) which an exact form, necessarily being closed, must also satisfy, we can set $U_{1}=Q \Omega_{1}+d_{1} \Omega_{0}+\delta \Omega_{2}$ with some $\Omega_{2}$. Referring to (5.34), this in turn means that $U_{0}+U_{1}$ is $\hat{Q}$-exact up to the degree in question. In an entirely similar manner, one can successively construct $U_{n}$ in the form $U_{n}=Q \Omega_{n}+d_{1} \Omega_{n-1}+d_{2} \Omega_{n-2}+\delta \Omega_{n+1}$ and hence finds $U=\sum_{n} U_{n}=\hat{Q} \Omega$, where $\Omega \equiv \sum_{n} \Omega_{n}$

The converses to these statements are much easier to prove. To show that $\hat{Q}$-closed $\Rightarrow Q$-closed, let $U=\sum_{n} U_{n}$ be $\hat{Q}$-closed and consider the projection map $U \rightarrow U_{0}$. Then, $U_{0}$ is $Q$-closed since it satisfies $Q U_{0}+\delta U_{1}=0$. Similarly, if we let $U=\sum_{n} U_{n}=\hat{Q} \Omega=$ $\sum_{n} \hat{Q} \Omega_{n}$, then $U_{0}$ is $Q$-exact as it satisfies $U_{0}=Q \Omega_{0}+\delta \Omega_{1}$. This shows $\hat{Q}$-exact $\Rightarrow$ $Q$-exact, and the entire proof of the equivalence of cohomologies is completed. 


\subsection{Action and energy-momentum tensor}

Now that we understand what fields are needed for our formalism, let us summarize their properties and write down the free action and the energy-momentum tensor. Besides the string coordinate $x^{\mu}$, we have the conjugate pairs $\left(\theta^{\alpha}, p_{\alpha}\right),\left(\lambda^{\alpha}, \omega_{\alpha}\right)$ and $\left(c_{I}, b_{I}\right)$ all carrying conformal dimensions $(0,1)$. The Euclidean action is given by

$$
S=\frac{1}{\pi} \int d^{2} z\left(\frac{1}{2} \partial x_{\mu} \bar{\partial} x^{\mu}+p_{\alpha} \bar{\partial} \theta^{\alpha}+\omega_{\alpha} \bar{\partial} \lambda^{\alpha}+b^{\mu} \bar{\partial} c_{\mu}\right),
$$

where we used the semi-covariant notations for the ghosts. The associated the energymomentum tensor is

$$
\begin{aligned}
T & =-\frac{1}{2} \partial x^{\mu} \partial x_{\mu}-p_{\alpha} \partial \theta^{\alpha}-\omega_{\alpha} \partial \lambda^{\alpha}-b^{\mu} \partial c_{\mu} \\
& =-\frac{1}{2} \Pi^{\mu} \Pi_{\mu}-d_{\alpha} \partial \theta^{\alpha}-\omega_{\alpha} \partial \lambda^{\alpha}-b^{\mu} \partial c_{\mu} .
\end{aligned}
$$

It is clear that the system forms a free CFT with vanishing central charge.

Since we have so far introduced only the minimum number of fermionic ghosts, they are not yet Lorentz invariant. A simple way to remedy this would be to introduce another five pairs of ghosts $\left(\tilde{c}_{I}, \tilde{b}_{I}\right)$ to promote our semi-covariant $c^{\mu}, b^{\mu}$ to genuine Lorentz vectors, as in [21]. Of course one needs to add additional ghosts to cancel the extra central charge produced by this revision. How this should be done, without spoiling the cohomology, to make the theory manifestly Lorentz covariant is under study and will be discussed in a separate publication [31.

\section{Vertex Operators for Massless Modes}

In order to compute the scattering amplitudes, one needs to construct the physical vertex operators. In this work, for simplicity we shall restrict ourselves to those for the super-Maxwell multiplet. Extension to the super-Yang-Mills case and to the closed string massless modes should be straightforward.

\subsection{Unintegrated vertex by homological perturbation}

Let us start with the construction of unitegrated vertex operator, to be denoted by $U$. With pure spinor constraints, it has been shown [9, 3] that the massless unintegrated vertex operator is given by

$$
U_{0}=\lambda^{\alpha} A_{\alpha}(x, \theta),
$$


where $A_{\alpha}$ is the spinor superfield that satisfies the on-shell condition

$$
\left(\gamma^{\mu_{1} \mu_{2} \ldots \mu_{5}}\right)^{\alpha \beta} D_{\alpha} A_{\beta}=0
$$

As is well-known [26, one can derive the following important equations from this condition, to be frequently used in the subsequent analysis:

$$
\begin{aligned}
& \text { (i) } \quad D_{\alpha} A_{\beta}+D_{\beta} A_{\alpha}=-2 i \gamma_{\alpha \beta}^{\mu} B_{\mu}, \\
& \text { (ii) } D_{\alpha} B_{\mu}-\partial_{\mu} A_{\alpha}=-2 i\left(\gamma_{\mu}\right)_{\alpha \beta} W^{\beta}, \\
& \text { (iii) } D_{\alpha} W^{\beta}=-\frac{1}{4}\left(\gamma^{\mu \nu}\right)_{\alpha}^{\beta} F_{\mu \nu}, \\
& \text { (iv) } D_{\alpha} F_{\mu \nu}=2 i\left(\left(\gamma_{\mu} \partial_{\nu} W\right)_{\alpha}-\left(\gamma_{\nu} \partial_{\mu} W\right)_{\alpha}\right), \\
& \text { (v) } \gamma^{\mu} \partial_{\mu} W^{\alpha}=0, \\
& \text { (vi) } \partial^{\mu} F_{\mu \nu}=0 .
\end{aligned}
$$

From $(i) \sim(i i i)$, one finds

$$
\begin{aligned}
B_{\mu} & =\frac{i}{16} \gamma_{\mu}^{\alpha \beta} D_{\alpha} A_{\beta} \\
W^{\alpha} & =\frac{i}{20}\left(\gamma^{\mu}\right)^{\alpha \beta}\left(D_{\beta} B_{\mu}-\partial_{\mu} A_{\beta}\right), \\
F_{\mu \nu} & =\partial_{\mu} B_{\nu}-\partial_{\nu} B_{\mu} .
\end{aligned}
$$

In our formalism without pure spinor constraints, $U_{0}$ must be extended to include additional pieces contaning $c_{I}$ ghosts so that the total vertex operator $U$ satisfies $\hat{Q} U=$ 0 . The method of construction should already be clear from the cohomology analysis presented in the previous section. Namely, we will expand the dimension 0 operator $U$ in powers of $c_{I}$ in the form $U=\sum_{n=0}^{5} U_{n}$, where $U_{n}$ contains products of $n c_{I}$ 's, and impose $\hat{Q} U=0$ to fix the coefficient fields, up to a $\hat{Q}$-exact form. In this analysis, $d_{2}$ term in $\hat{Q}$ can be dropped since it produces no singularity in the OPE's with any other quantities involved. Thus, the master equation at degree $n$ takes the form

$$
Q U_{n}+\delta U_{n+1}+d_{1} U_{n-1}=0
$$

From the general cohomology analysis we know that a consistent solution exists at every degree. More concretely, this means that $Q U_{n}+d_{1} U_{n-1}$ is guranteed to be $\delta$-exact so that $U_{n+1}$ can be found by using the triviality of the $\delta$-homology.

Let us begin at degree 0 . The master equation in this case is

$$
Q U_{0}+\delta U_{1}=0
$$


Using the equation (6.3) we immediately get

$$
Q U_{0}=-i \Lambda^{\mu} B_{\mu}
$$

Note that $Q U_{0}$ vanishes with PS constraints $\Lambda^{\mu}=0$, as it should. Then (6.13) is easily solved to give

$$
U_{1}=-c^{\mu} B_{\mu}
$$

At degree 1, the basic equation takes the form

$$
Q U_{1}+\delta U_{2}+d_{1} U_{0}=0
$$

A simple calculation using (6.4) yields

$$
Q U_{1}+d_{1} U_{0}=-2 i c^{\mu}\left(\lambda \gamma_{\mu} W\right)
$$

We recognize that the expression on the RHS of (6.17) is identical to the one we encountered in Sec.4, with $\partial \theta^{\alpha}$ in place of $W^{\alpha}$, and can be rewritten as

$$
Q U_{1}+d_{1} U_{0}=-2 i \lambda_{+}^{-1} c^{\nu} \Lambda^{\mu} \overline{\mathcal{N}}_{I}^{\nu}\left(\gamma_{\mu} W\right)_{I}
$$

This is manifestly proportional to $\Lambda^{\mu}$ and hence can be easily written as $-\delta U_{2}$. In this way we obtain

$$
U_{2}=-\lambda_{+}^{-1} c^{\mu} c^{\nu} \overline{\mathcal{N}}_{I}^{\nu}\left(\gamma_{\mu} W\right)_{I}
$$

The construction at degree 2 is a little more involved. The equation to solve is

$$
Q U_{2}+\delta U_{3}+d_{1} U_{1}=0
$$

and again to get $U_{3}$ we must compute $Q U_{2}+d_{1} U_{1}$ and write it as a $\delta$-exact form. For this calculation, we need Eq.(6.5), the Fierz identity (4.18) as well as an identity $c^{\mu}\left(\gamma_{\mu} \gamma_{\nu \rho} \lambda\right)_{I}=\lambda_{+}^{-1} c^{\sigma} \overline{\mathcal{N}}_{J}^{\sigma} \Lambda^{\mu}\left(\gamma_{\mu \nu \rho}\right)_{I J}-\lambda_{+}\left(c_{\nu} \mathcal{N}_{I}^{\rho}-c_{\rho} \mathcal{N}_{I}^{\nu}\right)$, where $\left(\gamma_{\mu \nu \rho}\right)_{I J} \equiv\left\langle I\left|\Gamma_{\mu \nu \rho}\right| \tilde{J}\right\rangle=$ $64 \epsilon_{I J P Q R} \overline{\mathcal{N}}_{P}^{\mu} \overline{\mathcal{N}}_{Q}^{\nu} \overline{\mathcal{N}}_{R}^{\rho}$. In this way, we get

$$
Q U_{2}+d_{1} U_{1}=-\frac{1}{4} \lambda_{+}^{-2} \Lambda^{\mu} c^{\sigma} c^{\tau} \overline{\mathcal{N}}_{I}^{\sigma} \overline{\mathcal{N}}_{J}^{\tau}\left(\gamma_{\mu \nu \rho}\right)_{I J} F^{\nu \rho}
$$

As this is again proportional to $\Lambda^{\mu}$, it is now a simple matter to express the RHS of (6.21) in the form $-\delta U_{3}$ and we obtain

$$
U_{3}=\frac{i}{12} \lambda_{+}^{-2} c^{\mu} c^{\sigma} c^{\tau} \overline{\mathcal{N}}_{I}^{\sigma} \overline{\mathcal{N}}_{J}^{\tau}\left(\gamma_{\mu \nu \rho}\right)_{I J} F^{\nu \rho}
$$


The master equation at degree 3, i.e. $Q U_{3}+d_{1} U_{2}+\delta U_{4}=0$, can be analyzed in a similar manner. Omitting the details, we find

$$
U_{4}=\frac{i}{12} \lambda_{+}^{-3} c^{\mu_{1}} c^{\mu_{2}} c^{\mu_{3}} c^{\mu} \overline{\mathcal{N}}_{I_{1}}^{\mu_{1}} \overline{\mathcal{N}}_{I_{2}}^{\mu_{2}} \overline{\mathcal{N}}_{I_{3}}^{\mu_{3}}\left(\gamma_{\mu \nu \rho}\right)_{I_{1} I_{2}}\left(\gamma^{\nu} \partial^{\rho} W\right)_{I_{3}}
$$

Now at degree 4 , where we have to solve $Q U_{4}+d_{1} U_{3}+\delta U_{5}=0$, we found that $Q U_{4}+d U_{3}$ vanishes identically using the on-shell condition as well as the Bianchi identity for $F_{\mu \nu}$. Thus we get the simple result $U_{5}=0$. Then the final equation at degree 5 , which serves as the consistency condition, reduces to $d_{1} U_{4}=0$ and it can be checked that this indeed holds.

Summarizing, we have found that the unintegrated vertex operator $U$ for the superMaxwell multiplet is given, up to a $\hat{Q}$-exact form, by

$$
\begin{aligned}
U & =\sum_{n=0}^{4} U_{n} \\
U_{0} & =\lambda^{\alpha} A_{\alpha} \\
U_{1} & =-c^{\mu} B_{\mu} \\
U_{2} & =-\lambda_{+}^{-1} c^{\mu} c^{\nu} \overline{\mathcal{N}}_{I}^{\nu}\left(\gamma_{\mu} W\right)_{I} \\
U_{3} & =\frac{i}{12} \lambda_{+}^{-2} c^{\mu} c^{\sigma} c^{\tau} \overline{\mathcal{N}}_{I}^{\sigma} \overline{\mathcal{N}}_{J}^{\tau}\left(\gamma_{\mu \nu \rho}\right)_{I J} F^{\nu \rho} \\
U_{4} & =\frac{i}{12} \lambda_{+}^{-3} c^{\mu_{1}} c^{\mu_{2}} c^{\mu_{3}} c^{\mu} \overline{\mathcal{N}}_{I_{1}}^{\mu_{1}} \overline{\mathcal{N}}_{I_{2}}^{\mu_{2}} \overline{\mathcal{N}}_{I_{3}}^{\mu_{3}}\left(\gamma_{\mu \nu \rho}\right)_{I_{1} I_{2}}\left(\gamma^{\nu} \partial^{\rho} W\right)_{I_{3}} .
\end{aligned}
$$

Evidently, $U$ is not manifestly Lorentz invariant. However, since the cohomology of $\hat{Q}$ is equivalent to that of $Q$ with pure spinor constraints, and since the latter has been shown to respect Lorentz covariance [25], it should be possible to extend our formalism, with additional ghosts, to make the Lorentz covariance manifest.

\subsection{Relation to Virasoro operator and construction of integrated vertex}

Having obtained the unitegrated vertex, our next task is to construct the integrated vertex operator, to be denoted by $\int[d z] V(z)$, which is required for computation of $n$ point amplitudes with $n \geq 4$. $V(z)$ of dimension 1 is characterized by the equation

$$
\hat{Q} V(z)=\partial U(z)
$$

so that $\int[d z] V(z)$ is annihilated by $\hat{Q}$. Besides the freedom of adding a $\hat{Q}$-exact term $\hat{Q} \Sigma$, $V$ has the ambiguity inherited from the change $U \rightarrow U+\hat{Q} \Omega$, which amounts to adding the total derivative $\partial \Omega$. 


\subsubsection{Construction by homological perturbation}

One way to construct $V$ is to make use of the scheme of homological perturbation, just as in the case of the unintegrated vertex. Decomposing (6.30) according to the degree, we get the master equation

$$
\begin{aligned}
& V=\sum_{n=n_{\min }}^{5} V_{n}, \\
& \partial U_{n}-Q V_{n}-d_{1} V_{n-1}-d_{2} V_{n-2}=\delta V_{n+1},
\end{aligned}
$$

where $n_{\min }$ is the lowest degree allowed for $V_{n}$. Contrary to the case of $U, n_{\min }$ can be as low as -1 since $V$ is of dimension 1 and a structure like $b^{\mu} X_{\mu}$ of degree -1 is possible. As it will be clear shortly, there are two types of solutions, related by a $\hat{Q}$-exact term, with either $n_{\text {min }}=-1$ or $n_{\text {min }}=0$.

Let us briefly discuss the $n_{\min }=0$ case, which gives a direct extension of the conventional Berkovits vertex [9, 3] given by

$$
V_{0}^{B}=\partial \theta^{\alpha} A_{\alpha}+\Pi^{\mu} B_{\mu}+d_{\alpha} W^{\alpha}+\frac{1}{2} L_{(\lambda)}^{\mu \nu} F_{\mu \nu},
$$

where $L_{(\lambda)}^{\mu \nu}$ is the Lorentz generator for the $(\lambda, \omega)$ sector:

$$
L_{(\lambda)}^{\mu \nu}=-\frac{1}{2}\left(\omega \gamma^{\mu \nu} \lambda\right) .
$$

With the PS constraints, this vertex satisfies the $n=0$ part of the master equation (6.32)

$$
\partial U_{0}-Q V_{0}=\delta V_{1}
$$

with $V_{1}=0$. When the PS constraints are removed, however, this no longer holds and one gets $\partial U_{0}-Q V_{0}^{B}=i \Lambda^{\mu}\left(\omega \partial_{\mu} W\right)$. The apparent problem is that this cannot possibly be written as $\delta V_{1}$ since the fact that $Q \delta V_{0}^{B} \neq 0$ contradicts the consistency equation obtained upon acting $\delta$ on (6.35). The solution to this problem turned out to be that one can modify $V_{0}^{B} \rightarrow V_{0}$ in such a way that $V_{0}$ satisfies both $\delta V_{0}=0$ and (6.35) simultaneously, with an appropriate $V_{1}$.

Once this is achieved, the rest of the construction is straightforward thanks to the triviality of $\delta$-homology proved in Appendix B: By using the the set of equations (5.27) $\sim(5.33)$ following from the nilpotency of $\hat{Q}$, it is easy to show that the LHS of (6.32) is annihilated by $\delta$ and hence can be written as $\delta V_{n+1}$, from which one can read off $V_{n+1}$. However, we shall not give the details of this procedure because there exists a conceptually superior way to construct $V$ with much less effort, to which we now turn. 


\subsection{2 "b-ghost", Virasoro operator and construction of $V$}

It is well-known that the action of the worldsheet derivative $\partial$ is implemented by that of $L_{-1}=\int[d z] T(z)$, where $T(z)$ is the energy-momentum tensor, given in our case by (5.40). Now suppose we can find the "b-ghost" field $B(z)$ such that

$$
T(z)=\hat{Q} B(z) \equiv\{\hat{Q}, B(z)\} .
$$

Then, if we define $\mathcal{B} \equiv \int[d z] B(z)$, we have $L_{-1}=\hat{Q} \mathcal{B}$ and hence acting on the unintegrated vertex $U$ one gets

$$
\partial U=(\hat{Q} \mathcal{B}) U=\hat{Q}(\mathcal{B} U)+\mathcal{B}(\hat{Q} U)=\hat{Q}(\mathcal{B} U),
$$

which is nothing but the defining equation for the integrated vertex $V$. Therefore we can construct $V$ simply as

$$
V=\mathcal{B} U
$$

A gratifying fact is that one can find such $B(z)$ in a remarkably simple form. It consists of degree -1 and 0 pieces and is given by

$$
\begin{aligned}
B(z) & =B_{-1}(z)+B_{0}(z), \\
B_{-1} & =-b^{\mu} \Pi_{\mu}, \quad B_{0}=-\omega_{\alpha} \partial \theta^{\alpha} .
\end{aligned}
$$

Notice that $B(z) B(w)=-b_{I}(z) b_{J}(w) \overline{\mathcal{N}}_{I}^{\mu} \overline{\mathcal{N}}_{J}^{\mu} /(z-w)^{2}=0$, which is a desired property.

Let us demonstrate that indeed $\hat{Q} B(z)=\left(\delta+Q+d_{1}+d_{2}\right) B(z)=T(z)$. First it is almost trivial to show that $Q B_{0}(z)=\partial \theta^{\alpha} d_{\alpha}-\omega_{\alpha} \partial \lambda^{\alpha}$ and $d_{1} B_{-1}(z)=-\frac{1}{2} \Pi^{\mu} \Pi_{\mu}-b_{\mu} \partial c^{\mu}$, so that

$$
T(z)=Q B_{0}(z)+d_{1} B_{-1}(z) .
$$

Next, it is just as easy to prove $\delta B_{0}(z)+Q B_{-1}(z)=0$ and $\delta B_{-1}(z)=0$. The remaining OPE's require non-trivial calculations. They can be performed with the help of $U(5)$ decompositions of certain quantities, details of which are not particularly illuminating and hence are omitted. The results, however, are extremely simple and we get $d_{1} B_{0}(z)+$ $d_{2} B_{-1}(z)=0$ and $d_{2} B_{0}(z)=0$. Altogether the proof of $\hat{Q} B(z)=T(z)$ is completed.

Now the complete integrated vertex $V$ is succinctly given by $V=\left(\mathcal{B}_{-1}+\mathcal{B}_{0}\right) U$ and can be easily computed explicitly. The structures up to degree 0 turned out to be particularly intriguing. A simple calculation gives

$$
\begin{aligned}
V= & \left(\mathcal{B}_{-1}+\mathcal{B}_{0}\right) U=b^{\mu} \lambda^{\alpha} \partial_{\mu} A_{\alpha}+\partial \theta^{\alpha} A_{\alpha}+\frac{1}{2} \Pi^{\mu} B_{\mu}-b^{\mu} c^{\nu} F_{\mu \nu} \\
& +\widetilde{\mathcal{N}}^{\mu \nu} \Pi_{\mu} B_{\nu}+c^{\mu} b^{\nu} \partial_{\mu} B_{\nu}+V_{n \geq 1} .
\end{aligned}
$$


where we defined $\tilde{\mathcal{N}}^{\mu \nu} \equiv \frac{1}{2}\left(\overline{\mathcal{N}}_{I}^{\mu} \mathcal{N}_{I}^{\nu}-\overline{\mathcal{N}}_{I}^{\nu} \mathcal{N}_{I}^{\mu}\right)$. Evidently, $V$ starts out from dergree -1 and its structure at degree 0 is, surprisingly, rather different from the Berkovits vertex $V_{0}^{B}$ : Among other things, $d_{\alpha} W^{\alpha}+\frac{1}{2} L_{(\lambda)}^{\mu \nu} F_{\mu \nu}$ part is missing and the coefficient of the term $\Pi^{\mu} B_{\mu}$ is only a half.

The solution to this puzzle is provided by the freedom of adding $\hat{Q}$-exact terms. First one can show that the degree -1 part of $V$ above can be rewritten exactly as $b^{\mu} \lambda^{\alpha} \partial_{\mu} A_{\alpha}=$ $-\delta(\omega W)-Q\left(b^{\mu} B_{\mu}\right)$. This means that if we add to $V$ the terms $\hat{Q}\left((\omega W)+\left(b^{\mu} B_{\mu}\right)\right)$, the resultant vertex starts from degree 0. Now a rather remarkable fact is that $Q(\omega W)$ contained in $\hat{Q}(\omega W)$ gives

$$
Q(\omega W)=d_{\alpha} W^{\alpha}+\frac{1}{2} L_{(\lambda)}^{\mu \nu} F_{\mu \nu}
$$

which is exactly the structure we are looking for. Moreover the missing half of $\Pi^{\mu} B_{\mu}$ is supplied by $d_{1}\left(b^{\mu} B_{\mu}\right)$ in $\hat{Q}\left(b^{\mu} B_{\mu}\right)$. In this way the alternative vertex becomes

$$
\begin{aligned}
\tilde{V} & =V+\hat{Q}\left(\omega W+b^{\mu} B_{\mu}\right) \\
& =V_{0}^{B}-b^{\mu} c^{\nu} F_{\mu \nu}+\frac{1}{2} \partial \tilde{\mathcal{N}}^{\mu \nu} F_{\mu \nu}+\tilde{V}_{n \geq 1},
\end{aligned}
$$

which indeed contains precisely the Berkovits vertex $V_{0}^{B}$. As expected, this vertex coincides with the one constructed by the homological perturbation technique described previously.

Besides being extremely useful for the construction of $V$, the existence of a simple form of " $b$-ghost" we have uncovered would have far-reaching consequences. The relation to the Virasoro generator, which hitherto has been rather elusive in PS formalism, is now

clearly understood and the relation $T=\{\hat{Q}, B\}$ is known to be of prime importance for no-ghost theorem and loop calculations [24]. Study of its significance in our formalism is now under investigation and will be reported elsewhere 31].

\section{Summary and Discussions}

In this paper, we have presented a new extension of the Berkovits' pure spinor formalism for superstring, in which pure spinor constraints are removed in a rather natural and efficient way with a minimum number of ghost fields. As a summary, it should be helpful to make a list of characteristic features of our formalism.

- With all the constraints removed, the question of how to actually treat the constraints in quantization procedure has been exorcized. 
- The Hilbert space structure is clarified and it is possible to realize the peculiar hermiticity property of $\lambda^{\alpha}$ with the aid of a modified Fock space inner product.

- PS constraints are precisely captured without redundancy and this led to the new simple first class algebra.

- In constructing the nilpotent BRST-like charge, a minimum number of additional ghosts $\left(c_{I}, b_{I}\right)_{I=1 \sim 5}$ are required.

- Everything fits nicely into the scheme of homological perturbation theory and the proof of the equivalence of cohomologies as well as the construction of massless vertex operators are achieved in a systematic manner.

- A simple composite " $b$-ghost" field $B(z)$ is constructed which realizes the fundamental equation $T(z)=\{\hat{Q}, B(z)\}$ and the relation to the Virasoro operator is thereby clarified. As an application, construction of the integrated vertex is made extremely efficient.

Obviously there are many remaining problems to be investigated and clarified. (i) First and foremost, although the Lorentz invariance of the cohomology is assured, we would like to further extend our formalism, by introducing additional ghosts, and achieve manifest Lorentz covariance. (ii) The calculation of scattering amplitudes should be performed and the rules of computation should be derived. (iii) Further consequences of the important

relation $T(z)=\{\hat{Q}, B(z)\}$ should be investigated. (iv) Relation to the RNS formalism needs to be clarified. (v) There should be no problem in applying our formalism to a superparticle case. Application to a supermembrane, on the other hand, is expected to be non-trivial and interesting. (vi) Other obvious problems are the extension to the case of curved background and description of D-branes in our formalism. These and related matters are under investigation and results will be reported elswhere [31].

\section{Acknowledgment}

We are grateful to M. Naka for his interest and collaboration at an early stage of this work. Y.K. thanks M. Kato for a useful discussion. The research of Y.K. is supported in part by the Grant-in-Aid for Scientific Research (B) No. 12440060 from the Japan Ministry of Education, Science and Culture. 


\section{Appendix A: Conventions and Useful Formulas}

In this appendix, we collect our conventions and some useful formulas employed in the text.

\section{A.1. Spinors and $\Gamma$-matrices in real basis}

$32 \times 32 S O(9,1)$ Gamma matrices are denoted by $\Gamma^{\mu},(\mu=0,1, \ldots, 9)$ and obey the Clifford algebra $\left\{\Gamma^{\mu}, \Gamma^{\nu}\right\}=2 \eta^{\mu \nu}$. Our metric convention is $\eta^{\mu \nu}=(-,+,+, \ldots,+)$. The 10-dimensional chirality operator is taken to be $\bar{\Gamma}_{10}=\Gamma^{0} \Gamma^{1} \cdots \Gamma^{9}$ and it satisfies $\bar{\Gamma}_{10}^{2}=1$.

In the Majorana or real basis (R-basis for short), $\Gamma^{\mu}$ are all real and unitary. Within the R-basis, we define the Weyl basis to be the one in which $\bar{\Gamma}_{10}=\operatorname{diag}\left(1_{16},-1_{16}\right)$, where $1_{16}$ is the $16 \times 16$ unit matrix. In this basis, a general 32 -component spinor $\Lambda$ is written as $\Lambda=\left(\begin{array}{c}\lambda^{\alpha} \\ \lambda_{\alpha}\end{array}\right)$, where $\lambda^{\alpha}$ and $\lambda_{\alpha}$ are chiral and anti-chiral respectively, with $\alpha=1 \sim 16$. Correspondingly, $\Gamma^{\mu}$, which flips chirality, takes the structure

$$
\Gamma^{\mu}=\left(\begin{array}{cc}
0 & \left(\gamma^{\mu}\right)^{\alpha \beta} \\
\left(\gamma^{\mu}\right)_{\alpha \beta} & 0
\end{array}\right)
$$

where the $16 \times 16 \gamma$-matrices $\left(\gamma^{\mu}\right)^{\alpha \beta}$ and $\left(\gamma^{\mu}\right)_{\alpha \beta}$ are real symmetric and satisfy

$$
\left(\gamma^{\mu}\right)_{\alpha \beta}\left(\gamma^{\nu}\right)^{\beta \gamma}+\left(\gamma^{\nu}\right)_{\alpha \beta}\left(\gamma^{\mu}\right)^{\beta \gamma}=2 \eta^{\mu \nu} \delta_{\alpha}^{\gamma}
$$

In terms of $\gamma^{\mu}$, an often used Fierz identity is expressed as $\left(\gamma_{\mu}\right)_{\alpha \beta}\left(\gamma^{\mu}\right)_{\gamma \delta}+($ cyclic in $\alpha, \beta, \gamma)=$ 0 .

Anti-symmetrized products of $\gamma^{\mu}$ are defined in the following way. $\left(\gamma^{\mu \nu}\right)_{\beta}^{\alpha}$ and $\left(\gamma^{\mu \nu}\right)_{\beta}{ }^{\alpha}$ are defined as

$$
\begin{aligned}
&\left(\gamma^{\mu \nu}\right)_{\beta}^{\alpha}=\frac{1}{2}\left(\left(\gamma^{\mu}\right)^{\alpha \gamma}\left(\gamma^{\nu}\right)_{\gamma \beta}-\left(\gamma^{\nu}\right)^{\alpha \gamma}\left(\gamma^{\mu}\right)_{\gamma \beta}\right), \\
&\left(\gamma^{\mu \nu}\right)_{\beta}^{\alpha}=\frac{1}{2}\left(\left(\gamma^{\mu}\right)_{\beta \gamma}\left(\gamma^{\nu}\right)^{\gamma \alpha}-\left(\gamma^{\nu}\right)_{\beta \gamma}\left(\gamma^{\mu}\right)^{\gamma \alpha}\right)
\end{aligned}
$$

and they are related by $\left(\gamma^{\mu \nu}\right)_{\beta}^{\alpha}=-\left(\gamma^{\mu \nu}\right)_{\beta}{ }^{\alpha} . \quad \gamma^{\mu_{1} \mu_{2} \ldots \mu_{k}}$ 's are similarly defined. Antisymmetric products with odd number of $\gamma^{\prime}$ s have definite symmtery properties. $\gamma^{\mu}$ and $\gamma^{\mu_{1} \ldots \mu_{5}}$ are symmetric, while $\gamma^{\mu_{1} \mu_{2} \mu_{3}}$ is anti-symmetric. $\left(\gamma^{\mu_{1} \ldots \mu_{5}}\right)^{\alpha \beta}$ is self-dual in the sense $\left(\gamma^{\mu_{1} \ldots \mu_{5}}\right)^{\alpha \beta}=(1 / 5 !) \epsilon^{\mu_{1} \ldots \mu_{5} \nu_{1} \ldots \nu_{5}}\left(\gamma_{\nu_{1} \ldots \nu_{5}}\right)^{\alpha \beta}$, where $\epsilon^{012 \ldots 9} \equiv 1$. Similarly, $\left(\gamma^{\mu_{1} \ldots \mu_{5}}\right)_{\alpha \beta}$ is antiself-dual.

Finally, we note that Lorentz covarint spinor bilinears of $\Lambda=\left(\begin{array}{c}\lambda^{\alpha} \\ \lambda_{\alpha}\end{array}\right)$ and $\Psi=$ 
$\left(\begin{array}{c}\psi^{\alpha} \\ \psi_{\alpha}\end{array}\right)$ can be constructed, without the use of complex conjugation, as

$$
\begin{aligned}
\Lambda^{T} C \Psi & =\lambda^{\alpha} \psi_{\alpha}-\lambda_{\alpha} \psi^{\alpha}, \\
\Lambda^{T} C \Gamma^{\mu} \Psi & =\lambda^{\alpha} \gamma_{\alpha \beta}^{\mu} \psi^{\beta}-\lambda_{\alpha}\left(\gamma^{\mu}\right)^{\alpha \beta} \psi_{\beta}, \\
\Lambda^{T} C \Gamma^{\mu \nu} \Psi & =\lambda^{\alpha}\left(\gamma^{\mu \nu}\right)_{\alpha}{ }^{\beta} \psi_{\beta}-\lambda_{\alpha}\left(\gamma^{\mu \nu}\right)^{\alpha}{ }_{\beta} \psi^{\beta}, \quad \text { etc },
\end{aligned}
$$

where $C$ is the charge conjugation matrix.

\section{A.2. $U(5)$ basis}

It is well-known that the spinor representations for $S O(9,1)$ and $S O(10)$ can be conveniently constructed with the use of 5 pairs of fermionic oscillators $\left(b_{I}, b_{I}^{\dagger}\right)$ satisfying the anti-commutation relations $\left\{b_{I}, b_{J}^{\dagger}\right\}=\delta_{I J}$. States are built upon the oscillator vacuum, to be denoted by $|+\rangle$, annihilated by all the $b_{I}$ 's. It is clear that the anti-commutation relations are invariant under the action of $U(5)$, where $b_{I}$ and $b_{I}^{\dagger}$ transform repsectively as 5 and $\overline{5}$ (or $\overline{5}$ and 5 , depending on one's convention). $\Gamma^{\mu}$ matrices can then be regarded as linear operators in this Fock space and in the case of $S O(10)$ they are identified as

$$
\Gamma^{2 I}=\frac{1}{i}\left(b_{I}-b_{I}^{\dagger}\right), \quad \Gamma^{2 I-1}=b_{I}+b_{I}^{\dagger}, \quad I=1 \sim 5 .
$$

Since the $S O(9,1)$ case is easily recovered by setting $\Gamma^{0}=i \Gamma^{10}=b_{0}-b_{0}^{\dagger}$, where $\left(b_{0}, b_{0}^{\dagger}\right) \equiv$ $\left(b_{5}, b_{5}^{\dagger}\right)$, we will use $S O(10)$ notations.

The states built upon $|+\rangle$ and their conjugates are defined as

$$
\begin{aligned}
\left|I_{1} I_{2} \ldots I_{k}\right\rangle & \equiv b_{I_{1}}^{\dagger} \cdots b_{I_{k}}^{\dagger}|+\rangle, \\
\left\langle I_{1} I_{2} \ldots I_{k}\right| & \equiv\langle+| b_{I_{k}} b_{I_{k-1}} \cdots b_{I_{1}} .
\end{aligned}
$$

Further, we define

$$
\begin{aligned}
|-\rangle & \equiv b_{1}^{\dagger} b_{2}^{\dagger} \ldots b_{5}^{\dagger}|+\rangle=\frac{1}{5 !} \epsilon_{I_{1} I_{2} \ldots I_{5}} b_{I_{1}}^{\dagger} b_{I_{2}}^{\dagger} \ldots b_{I_{5}}^{\dagger}|+\rangle, \\
\left|\tilde{I}_{1} \ldots \tilde{I}_{k}\right\rangle & \equiv \frac{1}{(5-k) !} \epsilon_{I_{1} \ldots I_{k} J_{k+1} \ldots J_{5}}\left|J_{k+1} \ldots J_{5}\right\rangle
\end{aligned}
$$

and their corresponding conjugates, where $\epsilon_{12345} \equiv 1$. These states satisfy the orthonormality relations

$$
\begin{aligned}
\langle+\mid+\rangle & =\langle-\mid-\rangle=1 \\
\left\langle I_{1} \ldots I_{k} \mid J_{1} \ldots J_{k}\right\rangle & =\left\langle\tilde{I}_{1} \ldots \tilde{I}_{k} \mid \tilde{J}_{1} \ldots \tilde{J}_{k}\right\rangle=\delta_{J_{1} \ldots J_{k}}^{I_{1} \ldots I_{k}}
\end{aligned}
$$


In this basis, chiral and anti-chiral spinors can be written as

$$
\begin{aligned}
\text { chiral: } & |\lambda\rangle & =\lambda_{+}|+\rangle+\frac{1}{2} \lambda_{I J}|I J\rangle+\lambda_{\tilde{I}}|\tilde{I}\rangle, \\
\text { anti-chiral: } & |\psi\rangle & =\psi_{-}|-\rangle+\frac{1}{2} \psi_{\tilde{I} \tilde{J}}|\tilde{I} \tilde{J}\rangle+\psi_{I}|I\rangle .
\end{aligned}
$$

We write the general components of chiral and anti-chiral spinors as $\lambda_{A}=\langle A \mid \lambda\rangle$ and $\psi_{\bar{A}}=\langle\bar{A} \mid \psi\rangle$.

The charge conjugation matrix in this basis is given by

$$
C=-i \Gamma^{2} \Gamma^{4} \Gamma^{6} \Gamma^{8} \Gamma^{10}=-\Gamma^{0} \Gamma^{2} \Gamma^{4} \Gamma^{6} \Gamma^{8} .
$$

Its action on the states is

$$
\begin{array}{lll}
C|+\rangle & =|-\rangle, \quad C|I J\rangle=-|\tilde{I} \tilde{J}\rangle, & C|\tilde{I}\rangle=|I\rangle, \\
C|-\rangle=-|+\rangle, & C|\tilde{I} \tilde{J}\rangle=|I J\rangle, & C|I\rangle=-|\tilde{I}\rangle .
\end{array}
$$

\section{Appendix B: Triviality of $\delta$-homology for degree $\geq 1$}

In this appendix, we give a proof of the triviality of the $\delta$-homology for degree $\geq 1$ in the general space of operators consisting of arbitrary number of $x^{\mu}, \theta^{\alpha}, \lambda^{\alpha}, \omega_{\alpha}, c_{I}, b_{I}$ and their worldsheet derivatives.

The action of $\delta=-i \int[d z] \Phi_{I}(z) b_{I}(z)$ in the sense of operator product can be represented more explicitly by the following three types of variational operations:

$$
\begin{aligned}
\delta & =\delta_{1}+\delta_{0} \\
\delta_{0} & =\delta_{0 a}+\delta_{0 b} \\
\delta_{1} & =-i \int[d z] \Phi_{I}(z) \frac{\delta}{\delta c_{I}(z)}, \\
\delta_{0 a} & =-i \int[d z] b_{I}(z) G_{I \alpha}(z) \frac{\delta}{\delta \omega_{\alpha}(z)}, \\
\delta_{0 b} & =-i \int[d z] G_{I \alpha}(z) \frac{\delta}{\delta c_{I}(z)} \frac{\delta}{\delta \omega_{\alpha}(z)} .
\end{aligned}
$$

Here the variational derivative $\delta / \delta a(z)$ is defined as $(\delta / \delta a(z)) a(w)=(z-w)^{-1}$ and $G_{I \alpha}(z)$ is the residue of the simple pole in the $\operatorname{OPE} \Phi_{I}(z) \omega_{\alpha}(w)=G_{I \alpha}(z) /(z-w) . \delta_{1}$ and $\delta_{0 a}$ effect the single contraction with $b_{I}(z)$ and $\Phi_{I}(z)$ respectively and $\delta_{0 b}$ represents the double contraction. The subscripts 0 and 1 signify the number of $\Phi_{I}$, to be referred to as " $\Phi$-level", increased by the operation ${ }^{14}$. Actually, when $\delta_{1}$ acts on $\partial^{m} c_{I}$ it produces

\footnotetext{
${ }^{14}$ Alternatively, " $\Phi$-level" is the same as the number of $\lambda_{\tilde{I}}$.
} 
$\partial^{m} \Phi_{I}$, but the derivatives acting on $\Phi$ will be irrelevant in the counting of $\Phi$-level. Clearly $\delta_{1}, \delta_{0 a}$ and $\delta_{0 b}$ anticommute with each other and with themselves.

In particular, $\delta_{1}^{2}=0$ and hence one can define $\delta_{1}$-homology. We shall now show that the demonstration of the triviality of $\delta$-homology can be reduced to that of $\delta_{1}$-homology. Since any operator not containing $c_{I}$ (or its worldsheet derivative) is annihilated by $\delta_{1}$, $\delta_{1}$-homology is obviously non-trivial at degree 0 . Hence the following argument applies to operators with degree $\geq 1$.

Assume that $\delta_{1}$-homology has been shown to be trivial and let $\mathcal{H}_{N}$ be the space of operators at $\Phi$-level $N$ or lower, where $N$ can be arbitrarily large. Then, any operator $A \in \mathcal{H}_{N}$ can be written as

$$
A=\sum_{n=0}^{N} A_{n},
$$

where $A_{n}$ is at $\Phi$-level $n$. Now suppose $\delta A=0$. Decomposing this equation according to the $\Phi$-level, we have

$$
0=\delta_{0} A_{n}+\delta_{1} A_{n-1}, \quad 0 \leq n \leq N+1
$$

with $A_{n} \equiv 0$ for $n<0$ and $n>N$. At the highest $\Phi$-level, we have $\delta_{1} A_{N}=0$ and hence by the triviality assumption $A_{N}$ must be $\delta_{1}$-exact, namely it can be written as $A_{N}=\delta_{1} B_{N-1}$. Using this result, the closedness relation at $\Phi$-level $N-1$ becomes

$$
\begin{aligned}
0 & =\delta_{0} A_{N}+\delta_{1} A_{N-1}=\delta_{0} \delta_{1} B_{N-1}+\delta_{1} A_{N-1} \\
& =\delta_{1}\left(A_{N-1}-\delta_{0} B_{N-1}\right)
\end{aligned}
$$

which can be solved as $A_{N-1}=\delta_{0} B_{N-1}+\delta_{1} B_{N-2}$. Continuing in this fashion, we can easily obtain the result $A=\delta B$, where $B=\sum_{n=0}^{N-1} B_{n}$. This proves the triviality of the $\delta$-homology.

Thus it suffices to prove the triviality of the $\delta_{1}$-homology. Let $\mathcal{H}_{M, N}$ be the space of operators where the largest number of $\partial$ on $c_{I}$ is $M$ and the largest number of such factor, $\partial^{M} c_{I}$, is $N$. $M$ and $N$ can be arbitrarily large. Assume that we have proved the triviality of $\delta_{1}$ up to $\mathcal{H}_{M-1, *}$ where $*$ is arbitrary. A general operator $A_{M}$ in $\mathcal{H}_{M, N}$ can then be written as

$$
\begin{aligned}
A_{M} & =\sum_{n=0}^{N} A_{M, n} \\
A_{M, n} & =\partial^{M} c_{I_{1}} \partial^{M} c_{I_{2}} \cdots \partial^{M} c_{I_{n}} \tilde{A}_{I_{1} I_{2} \ldots I_{n}}
\end{aligned}
$$


where $\tilde{A}_{I_{1} I_{2} \ldots I_{n}}$ as well as $A_{M, 0}$ are in $\mathcal{H}_{M-1, *}$. Now suppose $i \delta_{1} A_{M}=0$. Since the only term in $i \delta_{1} A_{M}$ containing the largest number, $N$, of $\partial^{M} c_{I}$ 's is $(-1)^{N} \partial^{M} c_{I_{1}} \partial^{M} c_{I_{2}} \cdots \partial^{M} c_{I_{N}} \delta_{1} \tilde{A}_{I_{1} I_{2} \ldots I_{N}}$, it must vanish separately. This means $\delta_{1} \tilde{A}_{I_{1} I_{2} \ldots I_{N}}=0$. From our assumption, this can be solved as

$$
\tilde{A}_{I_{1} I_{2} \ldots I_{N}}=\delta_{1} \tilde{B}_{I_{1} I_{2} \ldots I_{N}}
$$

Therefore we can write

$$
\begin{aligned}
A_{M, N}= & \partial^{M} c_{I_{1}} \partial^{M} c_{I_{2}} \cdots \partial^{M} c_{I_{N}} \delta_{1} \tilde{B}_{I_{1} I_{2} \ldots I_{N}} \\
= & \delta_{1}\left((-1)^{N} \partial^{M} c_{I_{1}} \partial^{M} c_{I_{2}} \cdots \partial^{M} c_{I_{N}} \tilde{B}_{I_{1} I_{2} \ldots I_{N}}\right) \\
& \quad-(-1)^{N} N \partial^{M} \Phi_{I_{1}} \partial^{M} c_{I_{2}} \cdots \partial^{M} c_{I_{N}} \tilde{B}_{I_{1} I_{2} \ldots I_{N}} .
\end{aligned}
$$

The term in the second line is $\delta_{1}$ exact (and hence automatically closed) while the term in the third line has only $N-1 \partial^{M} c_{I}$ 's and can be absorbed into $A_{M, N-1}$. This means that effectively $A_{M, N}$ can be removed from our analysis. Repeating this argument, we can remove all the $\partial^{M} c_{I}$ factors. We will then be left with operators belonging to $\mathcal{H}_{M-1, *}$, for which the triviality of $\delta_{1}$ holds by assumption.

In this way, by mathematical induction, the problem is reduced to the case of the space $\mathcal{H}_{0, *}$, i.e. , for operators without any $\partial$ 's on $c_{I}$ 's. The proof in this case, however, is formally the same as the simple situation already described in the main text: The action of $\delta$ in that case is operationally identical to that of $\delta_{1}$ in the present more general situation and hence $\delta_{1} A=0$ implies $A=\delta_{1} B$, due essentially to the algebraic independence of $\Phi_{I}$. This completes the proof.

\section{References}

[1] P. Ramond, Phys. Rev. D3 (1971) 2415.

A. Neveu and J. H. Schwarz, Nucl. Phys. B31 (1971) 86.

[2] M. B. Green and J. H. Schwarz, Nucl. Phys. B181 (1981) 502; Phys. Lett. B136 (1984) 367; Nucl. Phys. B243 (1984) 285.

[3] W. Siegel, Nucl. Phys. B263 (1986) 93.

[4] E. R. Nissimov and S. J. Pacheva, Phys. Lett. B189 (1987) 57; R. E. Kallosh, Phys. Lett. B195 (1987) 369; L. Brink, M. Henneaux and C. Teitelboim, Nucl. Phys. B293 (1987) 505. 
[5] E. Sokatchev, Class. Quant. Grav. 4 (1987) 237; E. Nissimov, S. Pacheva and S. Solomon, Nucl. Phys. B296 (1988) 462; E. R. Nissimov and S. J. Pacheva, Phys. Lett. B202 (1988) 325. R. Kallosh and M. Rakhmanov, Nucl. Phys. B209 (1988) 233.

[6] W. Siegel, Phys. Lett. B203, 79 (1988); U. Lindstrom, M. Rocek, W. Siegel, P. van Nieuwenhuizen and A. E. van de Ven, J. Math. Phys. 31 (1990) 1761; A. Mikovic, M. Rocek, W. Siegel, P. van Nieuwenhuizen, J. Yamron and A. E. van de Ven, Phys. Lett. B235 (1990) 106; M. B. Green and C. M. Hull, Nucl. Phys. B344 (1990) 115; Mod. Phys. Lett. A5 (1990) 1399; R. Kallosh, Phys. Lett. B251, 134 (1990); E. A. Bergshoeff, R. Kallosh and A. Van Proeyen, Phys. Lett. B251 (1990) 128; F. Essler, E. Laenen, W. Siegel and J. P. Yamron, Phys. Lett. B254 (1991) 411; F. Essler, M. Hatsuda, E. Laenen, W. Siegel, J. P. Yamron, T. Kimura and A. Mikovic, Nucl. Phys. B364 (1991) 67; J. L. Vazquez-Bello, Int. J. Mod. Phys. A7 (1992) 4583; E. A. Bergshoeff, R. Kallosh and A. Van Proeyen, Class. Quant. Grav. 9 (1992) 321.

[7] D. P. Sorokin, V. I. Tkach and D. V. Volkov, Mod. Phys. Lett. A4 (1989) 901; D. P. Sorokin, V. I. Tkach, D. V. Volkov and A. A. Zheltukhin, Phys. Lett. B216 (1989) 302; N. Berkovits, Phys. Lett. B232, 184 (1989); N. Berkovits, Nucl. Phys. B358, 169 (1991); A. Galperin and E. Sokatchev, Phys. Rev. D46 (1992) 714; M. Tonin, Phys. Lett. B266 (1991) 312; Int. J. Mod. Phys. A7 (1992) 6013; F. Delduc, E. Ivanov and E. Sokatchev, Nucl. Phys. B384 (1992) 334; N. Berkovits, Nucl. Phys. B379, 96 (1992); F. Delduc, A. Galperin, P. S. Howe and E. Sokatchev, Phys. Rev. D47 (1993) 578.

[8] N. Berkovits, Nucl. Phys. B431 (1994) 258; N. Berkovits, Nucl. Phys. B420 (1994) 332; N. Berkovits and C. Vafa, Mod. Phys. Lett. A9 (1994) 653; Nucl. Phys. B433 (1995) 123; N. Berkovits, Phys. Lett. B457 (1999) 94.

[9] N. Berkovits, JHEP 0004 (2000) 018, hep-th/0001035.

[10] E. Cartan, Leçon sur la Theorie des Spineurs, Hermann, Paris, 1937. (The Theory of Spinors, MIT Press, 1966. )

[11] P. S. Howe, Phys. Lett. B258 (1991) 141; Addendum, ibid B259 (1991) 511.

[12] P. S. Howe, Phys. Lett. B273 (1991) 90.

[13] N. Berkovits, JHEP 0009 (2000) 046, hep-th/0006003.

[14] N. Berkovits and B. C. Vallilo, JHEP 0007 (2000) 015, hep-th/0004171.

[15] N. Berkovits, JHEP 0108 (2001) 026, hep-th/0104247.

[16] N. Berkovits, JHEP 0109 (2001) 016, hep-th/0105050. 
[17] N. Berkovits, JHEP 0209 (2002) 051, hep-th/0201151.

[18] I. Oda and M. Tonin, Phys. Lett. B 520 (2001) 398, hep-th/0109051; N. Berkovits and P. S. Howe, Nucl. Phys. B 635 (2002) 75, hep-th/0112160, N. Berkovits and V. Pershin, hep-th/0205154 G. Trivedi, Mod. Phys. Lett. A 17 (2002) 2239, hep-th/0205217.

[19] N. Berkovits, ICTP Lectures on Covariant Quantization of the Superstring, hepth/0209059.

[20] M. Matone, L. Mzzucato, I. Oda, D. Sorokin and M. Tonin, Nucl. Phys. B639 (2002) 182, hep-th/0206104.

[21] P. A. Grassi, G. Policastro, M. Porrati and P. van Nieuwenhuizen, JHEP 0210 (2002) 054, hep-th/0112162.

P. A. Grassi, G. Policastro and P. van Nieuwenhuizen, hep-th/0202123.

P. A. Grassi, G. Policastro and P. van Nieuwenhuizen, hep-th/0206216.

P. A. Grassi, G. Policastro and P. van Nieuwenhuizen, hep-th/0209026.

P. A. Grassi, G. Policastro and P. van Nieuwenhuizen, hep-th/0211095.

[22] M. J. Chesterman, hep-th/0212261.

[23] M. Henneaux and C. Teitelboim, Quantization of Gauge Systems, Princeton University Press, 1992.

[24] M. D. Freeman and D. I. Olive, Phys. Lett. B175, (1986) 151; Phys. Lett. B175 (1986) 155.

[25] N. Berkovits and O. Chandia, Phys. Lett. B514 (2001) 394, hep-th/0105149.

[26] E. Witten, Nucl. Phys. B266 (1986) 245; J. P. Harnad and S. Shnider, Commun. Math. Phys. 106 (1986) 183; B. E. Nilsson, Class. Quant. Grav. 3 (1986) L41.

[27] N. Berkovits and O. Chandia, JHEP 0208 (2002) 040, hep-th/0204121.

[28] D. Friedan, E. Martinec and S. Shenker, Nucl. Phys. B271 (1986) 93.

[29] Y. Aisaka and Y. Kazama, work in progress.

[30] I. A. Batalin and G. A. Vilkovisky, Phys. Lett. B69 (1977) 309.

[31] Y. Aisaka and Y. Kazama, work in progress. 